Supporting Information

\title{
Elaboration of Aggregated Polysulfide Phases: from Molecules to Large Clusters and Solid Phases
}

Jiewen Xiao ${ }^{1}$, Guangmin Zhou' ${ }^{2}$,Hetian Chen ${ }^{1}$, Xiang Feng ${ }^{1}$, Dominik Legut ${ }^{3}$, Yanchen Fan ${ }^{1}$, Tianshuai Wang ${ }^{1}$, Yi Cui ${ }^{2,4^{*}}$, Qianfan Zhang ${ }^{1 *}$

1. School of Materials Science and Engineering, Beihang University, Beijing 100191, P. R. China.

2. Department of Materials Science and Engineering, Stanford University, Stanford, California 94305, United States.

3. IT4Innovations \& Nanotechnology Centre, VSB-Technical University of Ostrava, 17.listopadu 15, Ostrava CZ-70833, Czech Republic.

4. Stanford Institute for Materials and Energy Sciences, SLAC National Accelerator Laboratory, 2575 Sand Hill Road, Menlo Park, California 94025, United States

*Corresponding authors: yicui@stanford.edu and qianfan@buaa.edu.cn. 


\section{Section SI. Computational Details}

First-principles calculations were performed using the Vienna ab-initio simulation package (VASP) ${ }^{1,2}$ in the framework of density functional theory (DFT) with projector augmented wave (PAW) pseudopotentials. ${ }^{3,4}$ The Perdew-Burke-Ernzerhof (PBE) was adopted to describe the exchange-correlation function based on the generalized gradient approximation (GGA). ${ }^{5}$ The energy cutoff for plane wave basis was set to $500 \mathrm{eV}$ in conjunction with the Gamma-centered Monkhorst-Pack scheme for k-point sampling. ${ }^{6}$ The convergence criterion of the total energy was set to $10^{-5} \mathrm{eV}$. A Gaussian smearing of $0.02 \mathrm{eV}$ was adopted for the electronic temperature to speed up the calculation convergence. For geometric optimizations, all ions were relaxed to a force tolerance less than $0.01 \mathrm{eV} / \AA$. To achieve more precise band gaps, HSE06 (Heyd-ScuseriaErnzerhof) hybrid functional ${ }^{7,8}$ was further applied in those phases with small band gaps predicted by traditional GGA-PBE scheme. Bader charge analysis ${ }^{9,10}$ was performed to obtain charge distribution in different polysulfide phases. To analyze bonding and antibonding states in detail, Crystal Occupation Hamilton Population (COHP) was applied through using LOBSTER. ${ }^{11-14}$ It has been repeatedly demonstrated that the van der Waals (vdW) force plays an important role in lithium-sulfur battery system, ${ }^{15}$ so we have adopted vdW-DF2 functional ${ }^{16,17}$ to describe this physical interaction. Other vdW functionals, including rev-PBE ${ }^{18}$, opt-PBE ${ }^{16,17}$, opt-B88 ${ }^{16}$ and opt-B86b ${ }^{16}$ for vdW-DF1 and rev-vdW-DF2, ${ }^{19}$ were also used for comparative study. Besides, similar to oxygen involved system, there are some errors when calculating the formation energy of sulfurbased systems. Therefore, we have adopted the corrected formation energy from the 
Materials Project website, ${ }^{20-23}$ which has applied MP anion correction through using Wang's method. ${ }^{24,25}$ To prove dynamic stabilities of discovered solid phases, we have used PHONOPY26 to simulation phonon spectra and phonon density of states.

To obtain the geometric structures of polysulfide clusters and solid phases, the structure prediction was performed using Crystal Structure Analysis by Particle Swarm Optimization (CALYPSO). ${ }^{27}$ For polysulfide clusters, we have searched for dimers, tetramers and even octameters for $\mathrm{Li}_{2} \mathrm{~S}$. Similarly, three dimensional solid phases of $\mathrm{Li}_{2} \mathrm{~S}_{\mathrm{n}}(2 \leq \mathrm{n} \leq 8)$ are all explored based on the same scheme. For each polysulfide cluster and solid phase, more than 10,000 structures are generated and computed, while the structures with lowest energy and thermodynamic stability are extracted.

The dissolution behavior of polysulfides are explored from both the aspects of thermodynamics and kinetics. On the aspect of thermodynamics, dissolved polysulfide species are simulated by placing polysulfides in the middle of multiple randomly placed molecules of pure DME/DOL/DMF/DMSO solvents and the whole structure undergoes the ab-initio molecular dynamics (AIMD) ${ }^{28,29}$ simulation for 8 ps in order to ensure the equilibrium is reached. During AIMD simulation, NVT ensemble at 300K was adopted with Nose mass parameter being set to 0.5 to control temperature oscillations. Then the stable configuration for the electrolyte system is picked out and fully relaxed to extract thermodynamic information. And to further evaluate thermodynamic stabilities among dissolved molecules, large clusters and solid polysulfides with different depth of lithiation, we have calculated the lithium chemical potential of polysulfides, which can be described as: 


$$
\mu_{L i}=\left[E_{L i m S n}-n \mu_{S 8}-m \mu_{L i}^{0}\right] / m
$$

Where $E_{L i m S n}, \mu_{S 8}$ and $\mu_{L i}^{0}$ denote the energy per $\mathrm{Li}_{\mathrm{m}} \mathrm{S}_{\mathrm{n}}$ unit, the chemical potential of $\alpha-\mathrm{S}_{8}$ and Li metal bulk phase, respectively. It is worth to note that, from the aspect of thermodynamics, the lower the lithium chemical potential, the better the stability.

AIMD was further applied to investigate the kinetic behavior of polysulfide species and their interaction with electrolytes. For stable $\alpha-\mathrm{Li}_{2} \mathrm{~S}_{6}$ phase, we have adopted (001) slab with the low surface energy while DOL molecules are randomly packed above the slab based on their densities $\left(\sim 1.06 \mathrm{~g} / \mathrm{cm}^{3}\right)$ in order to see whether the $\mathrm{Li}_{2} \mathrm{~S}_{6}$ slab can be sustained under the effect of solvents. Similar to $\mathrm{Li}_{2} \mathrm{~S}_{6}$, the dynamic behavior for (001) slab of $\mathrm{Li}_{2} \mathrm{~S}_{8}$ was also studied. Climbing image-nudged elastic band $(\mathrm{CI}-\mathrm{NEB})^{30}$ methods was performed to quantitatively evaluate the energy barriers of the dissolution process of $\mathrm{Li}_{2} \mathrm{~S}_{6}$.

Voltage evolution among different $\mathrm{Li}_{2} \mathrm{~S}_{\mathrm{n}}$ stages was calculated based on Nernst equation: $E=-\frac{\Delta G}{n F}=\frac{\Delta E^{\text {internal }}+P \Delta V-T \Delta S}{n F} .{ }^{31}$ Here, $\mathrm{E}$ is the average potential, while $\Delta$ $E^{\text {internal }}, \Delta V$ and $\Delta S$ are the difference of internal energy, volume and entropy corresponding to the reaction. $\Delta G$ is approximated by $\Delta E^{\text {internal }}$ due to negligible contribution from the other two terms. ${ }^{32}$ 
Section SII. Geometric structures of polysulfide clusters and solid phases

\section{Atomic configurations of clusters and solid phases}

a.

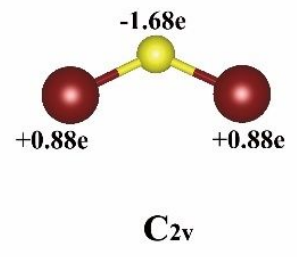

b.

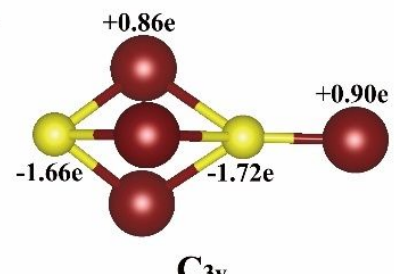

C3v c.

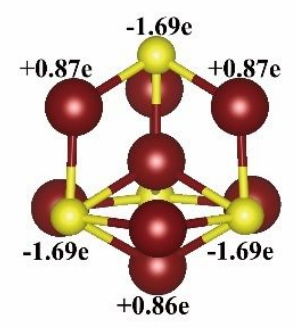

$\mathrm{C}_{3} \mathrm{v}$

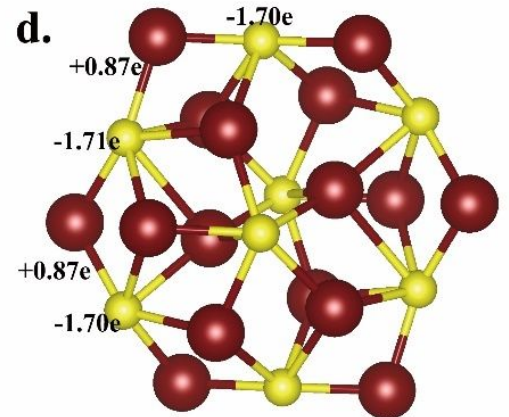

$\mathbf{C}_{\mathbf{i}}$ e.

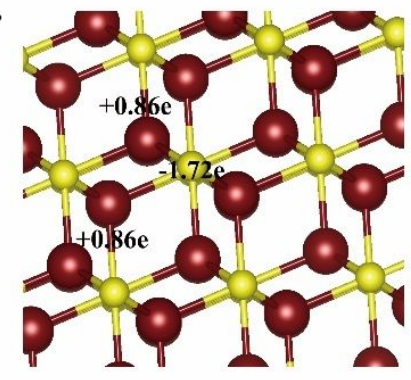

$\mathbf{F m} \overline{\mathbf{3}} \mathbf{m}$

Figure S1. Geometric structures of a. $\mathrm{Li}_{2} \mathrm{~S}$, b. $\left(\mathrm{Li}_{2} \mathrm{~S}\right)_{2}$, c. $\left(\mathrm{Li}_{2} \mathrm{~S}\right)_{4}$, d. $\left(\mathrm{Li}_{2} \mathrm{~S}\right)_{8}$ and e. $\mathrm{Li}_{2} \mathrm{~S}$ solid phase, whose point groups or space group are denoted below their atomic configurations. Charge distribution of typical atoms are also presented based on Bader charge analysis. 
a.

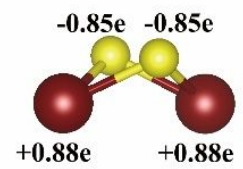

$\mathrm{C}_{2 \mathrm{v}}$

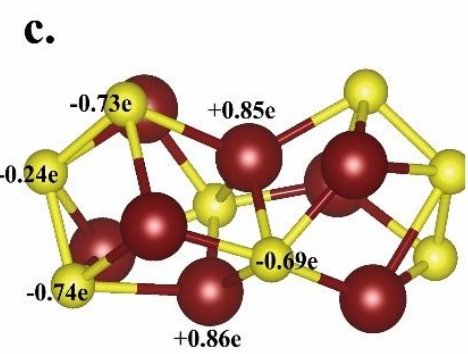

D2

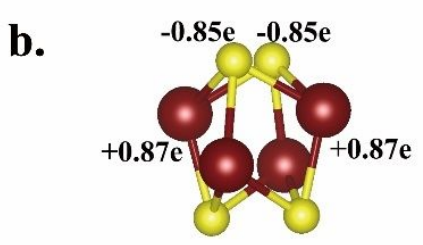

D2d

d.

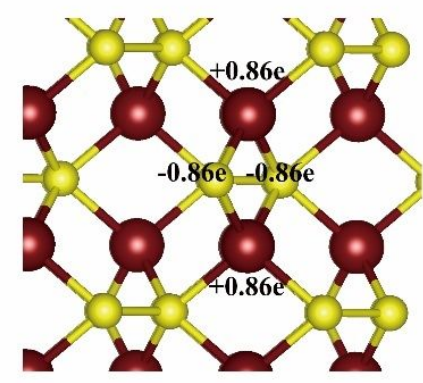

$\mathbf{P 4} 2 / \mathbf{m n m}$

Figure S2. Geometric structures of a. $\mathrm{Li}_{2} \mathrm{~S}_{2}$, b. $\left(\mathrm{Li}_{2} \mathrm{~S}_{2}\right)_{2}$, c. $\left(\mathrm{Li}_{2} \mathrm{~S}_{2}\right)_{4}$ and d. $\mathrm{Li}_{2} \mathrm{~S}_{2}$ solid phase, whose point groups or space group are denoted below their atomic configurations. Charge distribution of typical atoms are also presented based on Bader charge analysis. 
a.

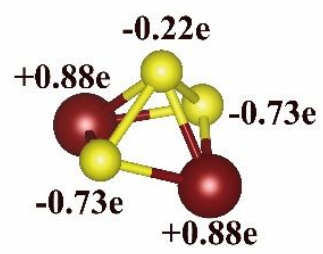

$\mathrm{C}_{2 \mathrm{v}}$

c.

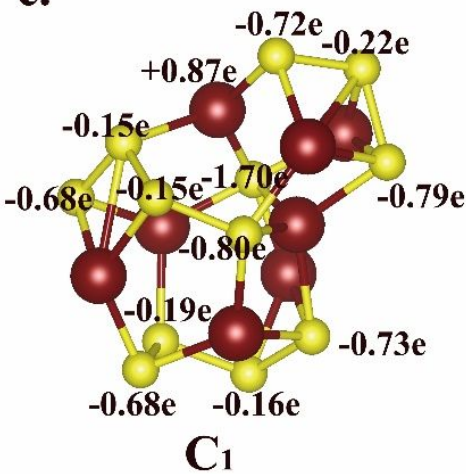

b.

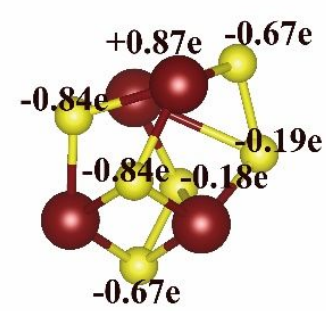

$\mathrm{C}_{2}$

d.

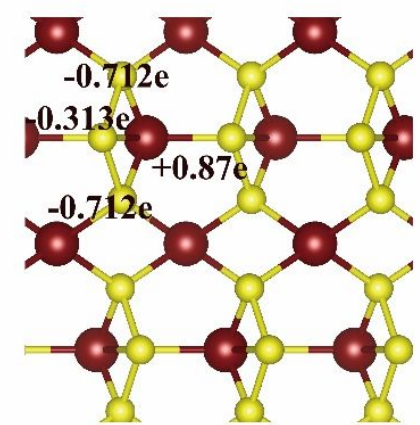

Ama2

Figure S3. Geometric structures of a. $\mathrm{Li}_{2} \mathrm{~S}_{3}$, b. $\left(\mathrm{Li}_{2} \mathrm{~S}_{3}\right)_{2}$, c. $\left(\mathrm{Li}_{2} \mathrm{~S}_{3}\right)_{4}$ and d. $\mathrm{Li}_{2} \mathrm{~S}_{3}$ solid phase, whose point groups or space group are denoted below their atomic configurations. Charge distribution of typical atoms are also presented based on Bader charge analysis. 
a.

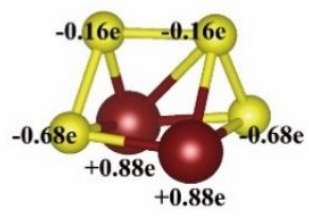

$\mathrm{C} 2$

c.

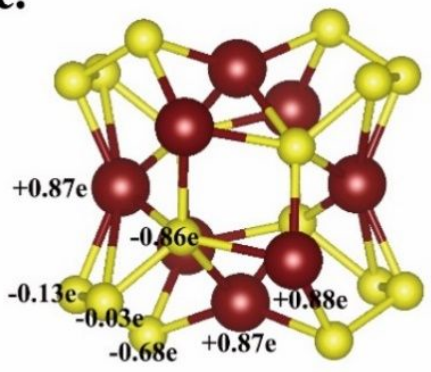

D2 b.

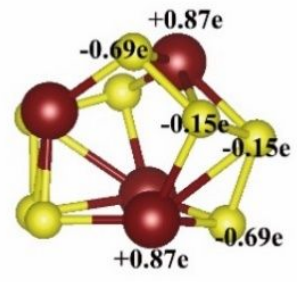

S4

d.

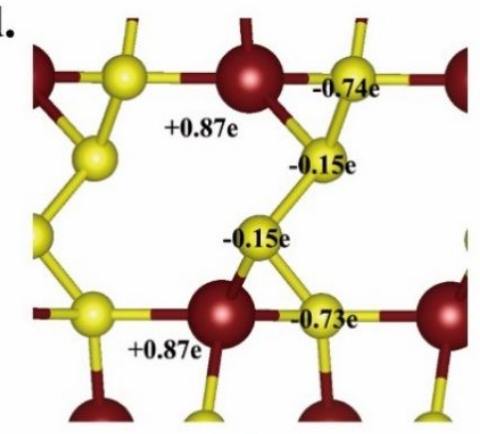

P1

Figure S4. Geometric structures of a. $\mathrm{Li}_{2} \mathrm{~S}_{4}$, b. $\left(\mathrm{Li}_{2} \mathrm{~S}_{4}\right)_{2}$, c. $\left(\mathrm{Li}_{2} \mathrm{~S}_{4}\right)_{4}$ and d. $\mathrm{Li}_{2} \mathrm{~S}_{4}$ solid phase, whose point groups or space group are denoted below their atomic configurations. Charge distribution of typical atoms are also presented based on Bader charge analysis. 
a.

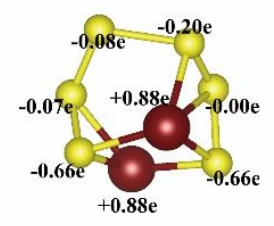

C1 b.

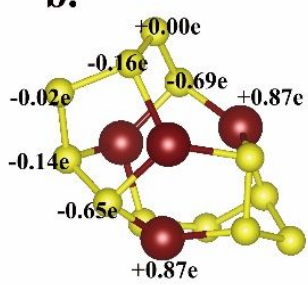

$\mathrm{C}_{1}$ c.

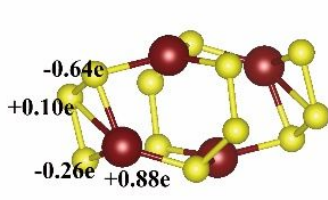

$\mathbf{C}_{\mathbf{i}}$

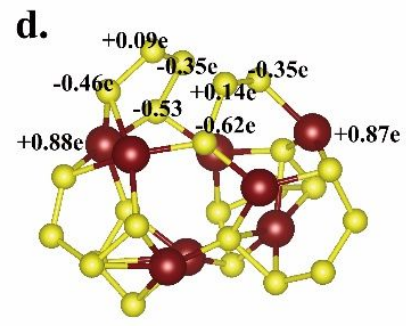

C1

e.

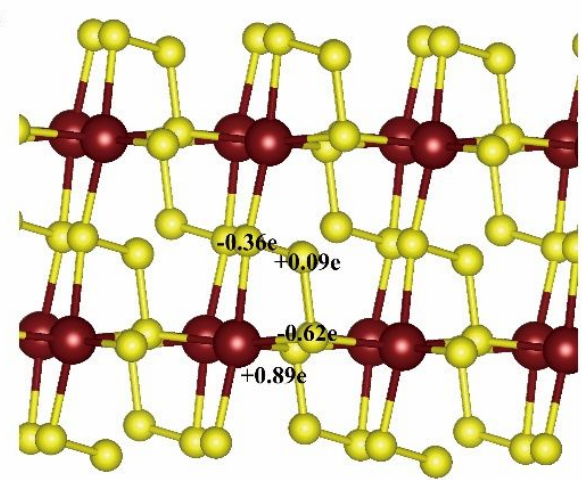

$\mathbf{P 2} 1$

f.

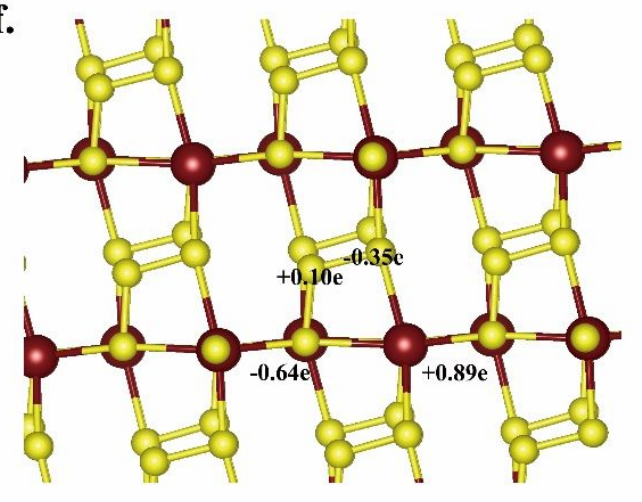

$\mathbf{P} \overline{\mathbf{1}}$

Figure S5. Geometric structures of a. $\mathrm{Li}_{2} \mathrm{~S}_{6}$, b. $\alpha-\left(\mathrm{Li}_{2} \mathrm{~S}_{6}\right)_{2}$, c. $\beta-\left(\mathrm{Li}_{2} \mathrm{~S}_{6}\right)_{2}$, d. $\left(\mathrm{Li}_{2} \mathrm{~S}_{6}\right)_{4}$, e. $\alpha-\mathrm{Li}_{2} \mathrm{~S}_{6}$ solid phase and f. $\beta-\mathrm{Li}_{2} \mathrm{~S}_{6}$ solid phase, whose point groups or space group are denoted below their atomic configurations. Charge distribution of typical atoms are also presented based on Bader charge analysis. $\alpha-\left(\operatorname{Li}_{2} \mathrm{~S}_{6}\right)_{2}$ is the most stable dimers of $\mathrm{Li}_{2} \mathrm{~S}_{6}$ while $\beta-\left(\mathrm{Li}_{2} \mathrm{~S}_{6}\right)_{2}$ is meta stable phases, being with $\sim 0.4 \mathrm{meV} /$ atom above $\alpha$ - $\left(\mathrm{Li}_{2} \mathrm{~S}_{6}\right)_{2}$ phase. 
a.

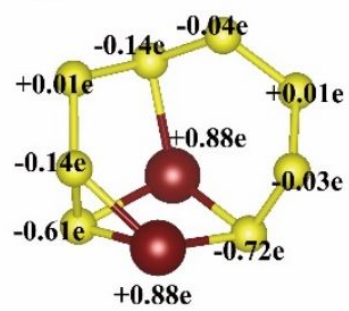

C1 b.

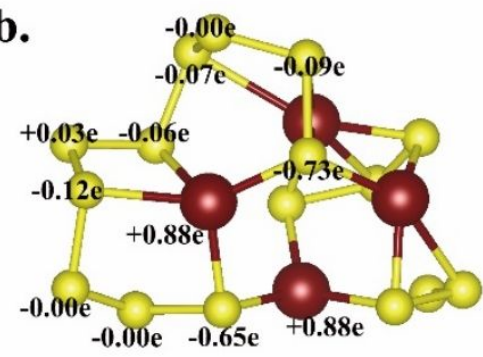

C1

c.

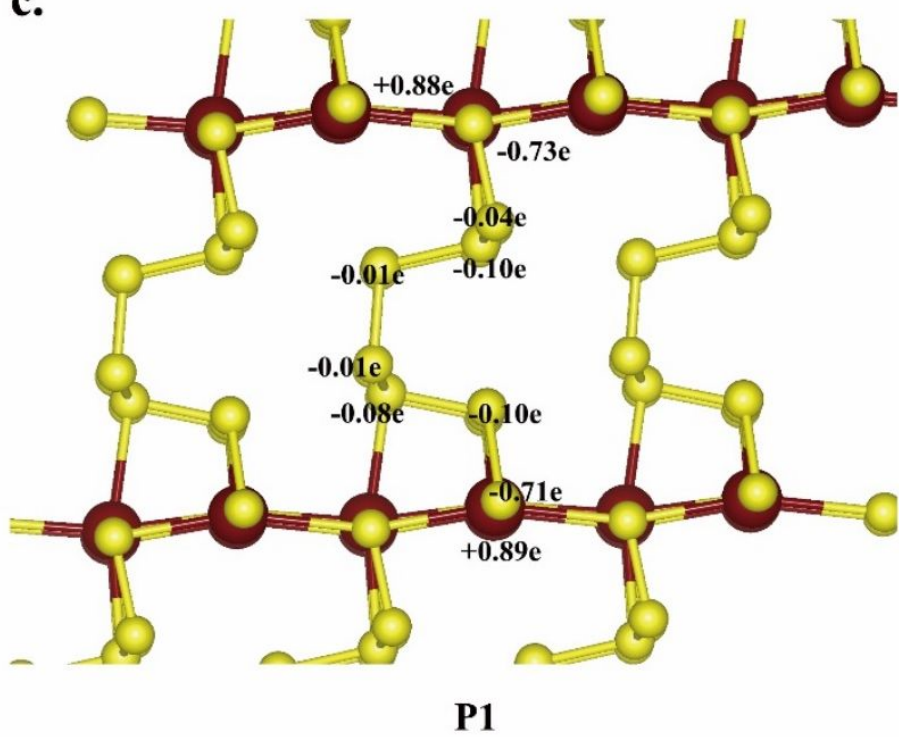

Figure S6. Geometric structures of a. $\mathrm{Li}_{2} \mathrm{~S}_{8}$, b. $\left(\mathrm{Li}_{2} \mathrm{~S}_{8}\right)_{2}$ and c. $\mathrm{Li}_{2} \mathrm{~S}_{8}$ solid phase, whose point groups or space group are denoted below their atomic configurations. Charge distribution of typical atoms are also presented based on Bader charge analysis. 
a.

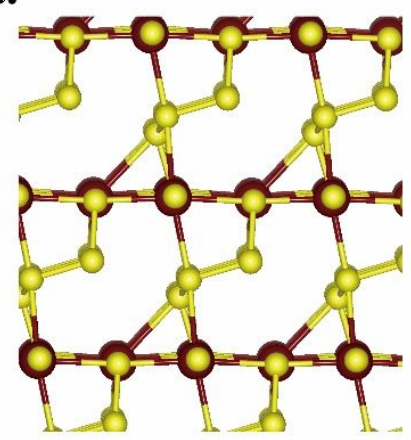

b.

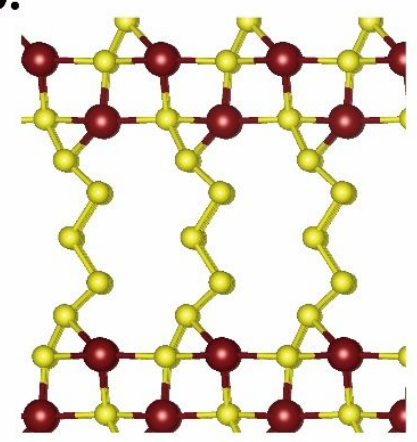

Figure S7. Geometric structures of a. solid state $\mathrm{Li}_{2} \mathrm{~S}_{5}$, b. solid state $\mathrm{Li}_{2} \mathrm{~S}_{7}$, which both sit above convex hull in phase diagram, thus being thermodynamically unstable.

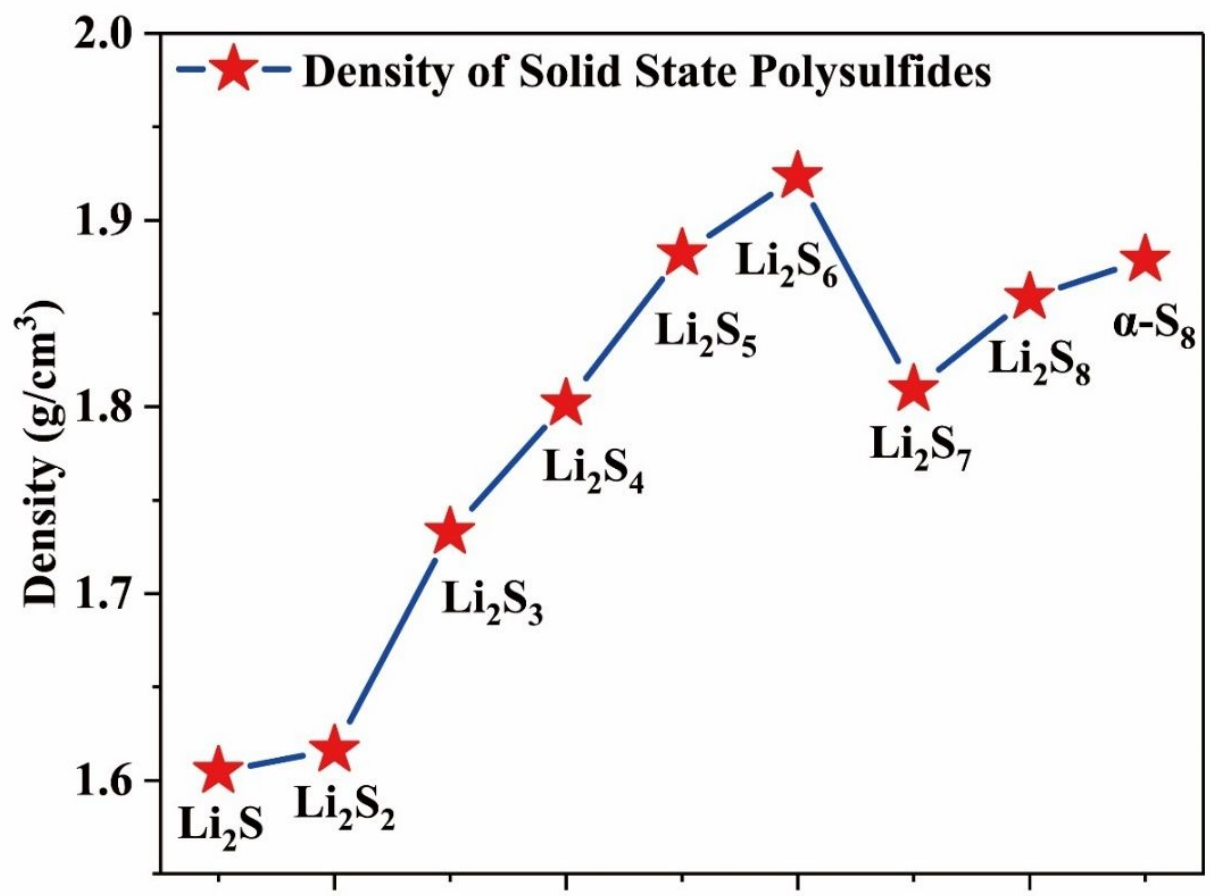

Figure S8. Evolution of densities of solid state polysulfides. 


\section{Phase Diagram}

a.

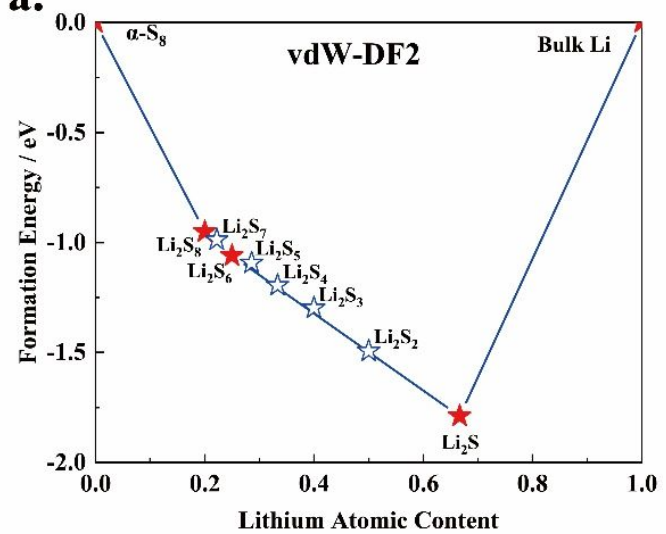

c.

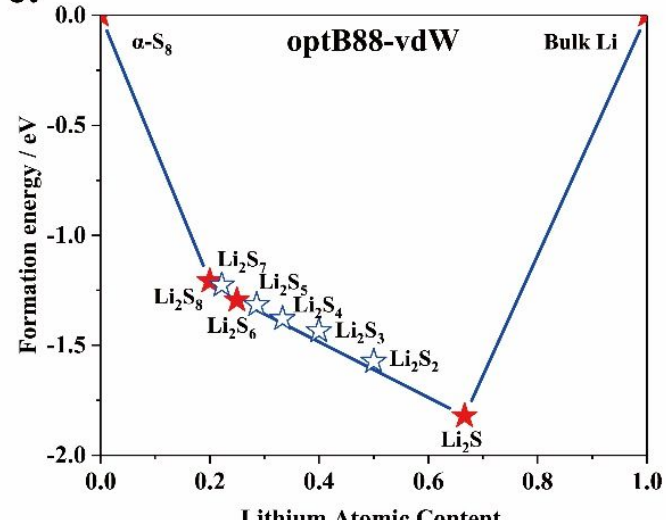

e.

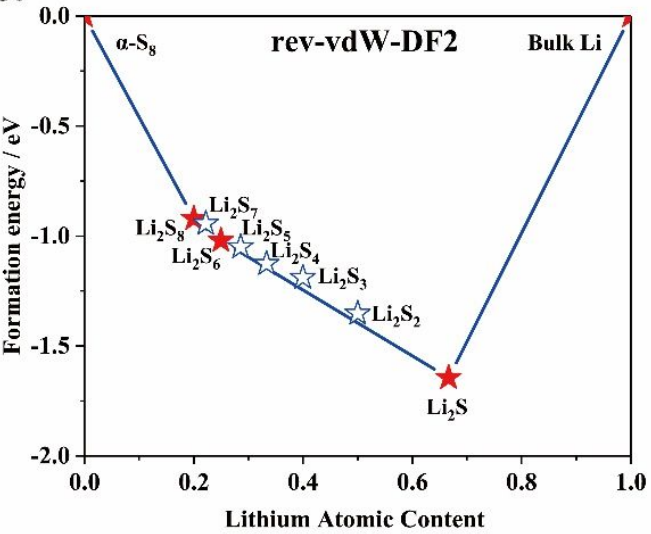

b.

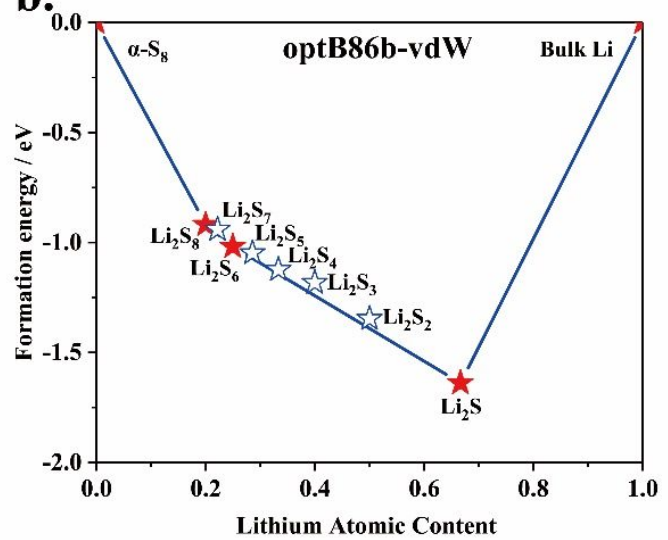

d.

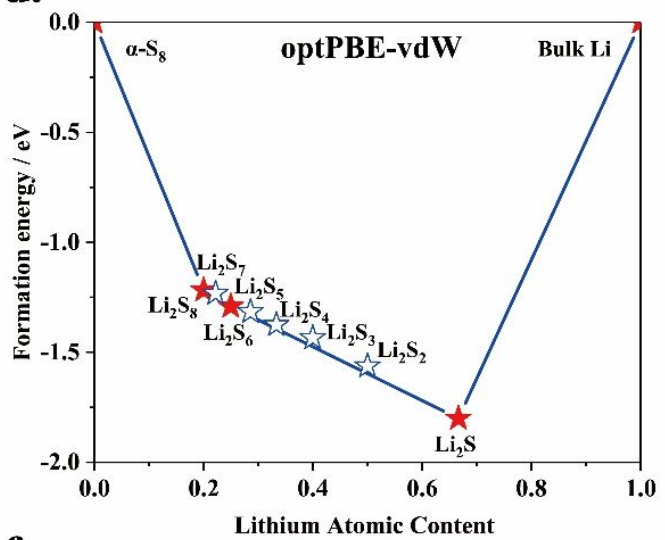

f.

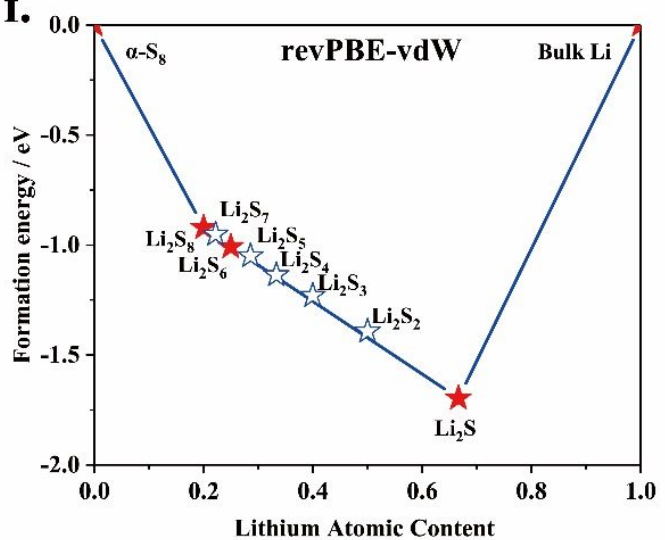

Figure S9. Calculated Phase diagram through using different types of vdW functional: a. vdW-DF2, b. optB86b, c. optB88, d. optPBE, e. rev-vdW-DF2 and f. revPBE 


\section{Detailed geometric information of polysulfide solid phases}

Table S1. Lattice parameters, space group and density of polysulfide solid phases.

\begin{tabular}{|c|c|c|c|c|c|c|c|c|}
\hline \multirow{2}{*}{$\begin{array}{c}\text { Solid } \\
\text { Phases }\end{array}$} & \multicolumn{6}{|c|}{ Lattice Parameters } & \multirow{2}{*}{$\begin{array}{l}\text { Space } \\
\text { Group }\end{array}$} & \multirow{2}{*}{$\begin{array}{l}\text { Density } \\
\left(\mathrm{g} / \mathrm{cm}^{3}\right)\end{array}$} \\
\hline & $a / \AA$ & $b / \AA$ & $c / \AA$ & $\alpha$ & $\beta$ & $\gamma$ & & \\
\hline $\mathrm{Li}_{2} \mathrm{~S}_{2}$ & 5.114 & 5.114 & 6.130 & 90.00 & 90.00 & 90.00 & 136 & 1.616 \\
\hline $\mathrm{Li}_{2} \mathrm{~S}_{3}$ & 7.249 & 4.896 & 11.889 & 90.00 & 90.22 & 90.00 & 40 & 1.733 \\
\hline $\mathrm{Li}_{2} \mathrm{~S}_{4}$ & 4.344 & 4.777 & 6.980 & 108.21 & 73.75 & 101.80 & 1 & 1.802 \\
\hline $\mathrm{Li}_{2} \mathrm{~S}_{5}$ & 5.333 & 5.306 & 5.555 & 97.93 & 98.62 & 91.71 & 1 & 1.882 \\
\hline$\alpha-\mathrm{Li}_{2} \mathrm{~S}_{6}$ & 5.278 & 7.985 & 8.531 & 90.00 & 97.77 & 90.00 & 4 & 1.923 \\
\hline$\beta-\mathrm{Li}_{2} \mathrm{~S}_{6}$ & 6.487 & 7.044 & 7.927 & 88.68 & 93.26 & 90.89 & 2 & 1.895 \\
\hline $\mathrm{Li}_{2} \mathrm{~S}_{7}$ & 4.559 & 4.544 & 11.156 & 99.43 & 99.37 & 101.56 & 1 & 1.809 \\
\hline $\mathrm{Li}_{2} \mathrm{~S}_{8}$ & 5.337 & 5.357 & 8.946 & 100.86 & 105.46 & 90.69 & 1 & 1.859 \\
\hline
\end{tabular}

Table S2. Atom positions and corresponding Bader charges of solid state $\mathrm{Li}_{2} \mathrm{~S}_{2}$.

\begin{tabular}{cccccc}
\hline & & \multicolumn{3}{c}{ Fractional Coordinates } & Bader \\
Element & Label & $\mathbf{X}$ & $\mathbf{y}$ & $\mathbf{Z}$ & Charge \\
\hline $\mathrm{S}$ & $\mathrm{S} 1$ & 0.849706 & 0.150294 & 0.500000 & $-0.865 \mathrm{e}$ \\
$\mathrm{S}$ & $\mathrm{S} 2$ & 0.150294 & 0.849706 & 0.500000 & $-0.863 \mathrm{e}$ \\
$\mathrm{S}$ & $\mathrm{S} 3$ & 0.349706 & 0.349706 & 0.000000 & $-0.865 \mathrm{e}$ \\
$\mathrm{S}$ & $\mathrm{S} 4$ & 0.650294 & 0.650294 & 1.000000 & $-0.863 \mathrm{e}$ \\
$\mathrm{Li}$ & $\mathrm{Li} 1$ & 0.500000 & 0.000000 & 0.250000 & $+0.864 \mathrm{e}$ \\
$\mathrm{Li}$ & $\mathrm{Li} 2$ & 1.000000 & 0.500000 & 0.750000 & $+0.864 \mathrm{e}$ \\
$\mathrm{Li}$ & $\mathrm{Li} 3$ & 0.500000 & 0.000000 & 0.750000 & $+0.864 \mathrm{e}$ \\
$\mathrm{Li}$ & $\mathrm{Li}$ & 1.000000 & 0.500000 & 0.250000 & $+0.864 \mathrm{e}$ \\
\hline
\end{tabular}


Table S3. Atom positions and corresponding Bader charges of solid state $\mathrm{Li}_{2} \mathrm{~S}_{3}$.

\begin{tabular}{|c|c|c|c|c|c|}
\hline \multirow[b]{2}{*}{ Element } & \multirow[b]{2}{*}{ Label } & \multicolumn{3}{|c|}{ Fractional Coordinates } & \multirow{2}{*}{$\begin{array}{l}\text { Bader } \\
\text { Charge }\end{array}$} \\
\hline & & $\mathbf{x}$ & $\mathbf{y}$ & $\mathbf{z}$ & \\
\hline $\mathrm{S}$ & S1 & 0.176768 & 0.867382 & 0.250000 & $-0.310 \mathrm{e}$ \\
\hline $\mathrm{S}$ & S2 & 0.823232 & 0.867382 & 0.750000 & $-0.310 \mathrm{e}$ \\
\hline $\mathrm{S}$ & S3 & 0.246255 & 0.130348 & 0.604768 & $-0.713 \mathrm{e}$ \\
\hline $\mathrm{S}$ & S4 & 0.753745 & 0.130348 & 0.395232 & $-0.713 e$ \\
\hline S & S5 & 0.753745 & 0.130348 & 0.104768 & $-0.713 \mathrm{e}$ \\
\hline $\mathrm{S}$ & S6 & 0.246255 & 0.130348 & 0.895232 & $-0.713 \mathrm{e}$ \\
\hline $\mathrm{S}$ & S7 & 0.676768 & 0.367382 & 0.250000 & $-0.310 \mathrm{e}$ \\
\hline $\mathrm{S}$ & S8 & 0.323232 & 0.367382 & 0.750000 & $-0.310 \mathrm{e}$ \\
\hline $\mathrm{S}$ & S9 & 0.746255 & 0.630348 & 0.604768 & $-0.713 \mathrm{e}$ \\
\hline $\mathrm{S}$ & S10 & 0.253745 & 0.630348 & 0.395232 & $-0.713 e$ \\
\hline S & S11 & 0.253745 & 0.630348 & 0.104768 & $-0.713 \mathrm{e}$ \\
\hline $\mathrm{S}$ & S12 & 0.746255 & 0.630348 & 0.895232 & $-0.713 \mathrm{e}$ \\
\hline $\mathrm{Li}$ & Li1 & 1.000000 & 0.392163 & 0.000000 & $+0.870 \mathrm{e}$ \\
\hline $\mathrm{Li}$ & Li2 & 0.000000 & 0.392163 & 0.500000 & $+0.870 \mathrm{e}$ \\
\hline $\mathrm{Li}$ & $\mathrm{Li} 3$ & 0.846107 & 0.807550 & 0.250000 & $+0.865 \mathrm{e}$ \\
\hline $\mathrm{Li}$ & Li4 & 0.153893 & 0.807550 & 0.750000 & $+0.865 \mathrm{e}$ \\
\hline $\mathrm{Li}$ & Li5 & 0.500000 & 0.892163 & 1.000000 & $+0.870 \mathrm{e}$ \\
\hline $\mathrm{Li}$ & Li6 & 0.500000 & 0.892163 & 0.500000 & $+0.870 \mathrm{e}$ \\
\hline $\mathrm{Li}$ & Li7 & 0.346107 & 0.307550 & 0.250000 & $+0.865 \mathrm{e}$ \\
\hline $\mathrm{Li}$ & Li8 & 0.653893 & 0.307550 & 0.750000 & $+0.865 \mathrm{e}$ \\
\hline
\end{tabular}


Table S4. Atom positions and corresponding Bader charges of solid state $\mathrm{Li}_{2} \mathrm{~S}_{4}$.

\begin{tabular}{cccccc}
\hline & & \multicolumn{3}{c}{ Fractional Coordinates } & Bader \\
Element & Label & $\mathbf{x}$ & $\mathbf{y}$ & $\mathbf{Z}$ & Charge \\
\hline $\mathrm{S}$ & $\mathrm{S} 1$ & 0.396689 & 0.235250 & 0.002779 & $-0.153 \mathrm{e}$ \\
$\mathrm{S}$ & $\mathrm{S} 2$ & 0.592523 & 0.607653 & 0.221570 & $-0.730 \mathrm{e}$ \\
$\mathrm{S}$ & $\mathrm{S} 3$ & 0.963642 & 0.229955 & 0.553016 & $-0.723 \mathrm{e}$ \\
$\mathrm{S}$ & $\mathrm{S} 4$ & 0.757337 & 0.992557 & 0.782151 & $-0.122 \mathrm{e}$ \\
$\mathrm{Li}$ & $\mathrm{Li} 1$ & 0.155261 & 0.864086 & 0.219475 & $+0.872 \mathrm{e}$ \\
$\mathrm{Li}$ & $\mathrm{Li} 2$ & 0.514158 & 0.475818 & 0.553388 & $+0.871 \mathrm{e}$ \\
\hline
\end{tabular}

Table S5. Atom positions and corresponding Bader charges of solid state $\mathrm{Li}_{2} \mathrm{~S}_{5}$.

\begin{tabular}{cccccc}
\hline & & \multicolumn{3}{c}{ Fractional Coordinates } & Bader \\
Element & Label & $\mathbf{X}$ & $\mathbf{y}$ & $\mathbf{Z}$ & Charge \\
\hline S & S1 & 0.140378 & 0.392209 & 0.692197 & $-0.710 \mathrm{e}$ \\
S & S2 & 0.701877 & 0.812035 & 0.289144 & $-0.119 \mathrm{e}$ \\
S & S3 & 0.622433 & 0.411029 & 0.175402 & $-0.149 \mathrm{e}$ \\
S & S4 & 0.630663 & 0.885725 & 0.656524 & $-0.704 \mathrm{e}$ \\
S & S5 & 0.224164 & 0.335309 & 0.065042 & $-0.090 \mathrm{e}$ \\
Li & Li1 & 0.650344 & 0.379857 & 0.677748 & $+0.883 \mathrm{e}$ \\
Li & Li2 & 0.134641 & 0.886825 & 0.659744 & $+0.889 \mathrm{e}$ \\
\hline
\end{tabular}


Table S6. Atom positions and corresponding Bader charges of solid state $\alpha-\mathrm{Li}_{2} \mathrm{~S}_{6}$.

\begin{tabular}{|c|c|c|c|c|c|}
\hline \multirow[b]{2}{*}{ Element } & \multirow[b]{2}{*}{ Label } & \multicolumn{3}{|c|}{ Fractional Coordinates } & \multirow{2}{*}{$\begin{array}{l}\text { Bader } \\
\text { Charge }\end{array}$} \\
\hline & & $\mathbf{x}$ & $\mathbf{y}$ & $\mathbf{z}$ & \\
\hline $\mathrm{S}$ & S1 & 0.999380 & 0.133797 & 0.219762 & $-0.364 \mathrm{e}$ \\
\hline $\mathrm{S}$ & S2 & 0.001115 & 0.190090 & 0.720167 & $-0.363 \mathrm{e}$ \\
\hline $\mathrm{S}$ & S3 & 0.000620 & 0.633797 & 0.780238 & $-0.364 \mathrm{e}$ \\
\hline $\mathrm{S}$ & S4 & 0.998885 & 0.690090 & 0.279833 & $-0.363 \mathrm{e}$ \\
\hline $\mathrm{S}$ & S5 & 0.471114 & 0.459461 & 0.022609 & $-0.618 \mathrm{e}$ \\
\hline $\mathrm{S}$ & S6 & 0.923405 & 0.964857 & 0.044029 & $+0.094 \mathrm{e}$ \\
\hline $\mathrm{S}$ & S7 & 0.529840 & 0.363927 & 0.478361 & $-0.617 \mathrm{e}$ \\
\hline $\mathrm{S}$ & S8 & 0.075585 & 0.858955 & 0.455615 & $+0.094 \mathrm{e}$ \\
\hline $\mathrm{S}$ & S9 & 0.076595 & 0.464857 & 0.955971 & $+0.094 \mathrm{e}$ \\
\hline $\mathrm{S}$ & S10 & 0.528886 & 0.959461 & 0.977391 & $-0.618 \mathrm{e}$ \\
\hline S & S11 & 0.924415 & 0.358955 & 0.544385 & $+0.094 \mathrm{e}$ \\
\hline S & S12 & 0.470160 & 0.863927 & 0.521639 & $-0.617 \mathrm{e}$ \\
\hline $\mathrm{Li}$ & Li1 & 0.490889 & 0.199114 & 0.203302 & $+0.887 \mathrm{e}$ \\
\hline $\mathrm{Li}$ & Li2 & 0.492221 & 0.123009 & 0.702639 & $+0.886 \mathrm{e}$ \\
\hline $\mathrm{Li}$ & Li3 & 0.509111 & 0.699114 & 0.796697 & $+0.887 \mathrm{e}$ \\
\hline $\mathrm{Li}$ & Li4 & 0.507779 & 0.623009 & 0.297361 & $+0.886 \mathrm{e}$ \\
\hline
\end{tabular}


Table S7. Atom positions and corresponding Bader charges of solid state $\beta-\mathrm{Li}_{2} \mathrm{~S}_{6}$.

\begin{tabular}{|c|c|c|c|c|c|}
\hline \multirow[b]{2}{*}{ Element } & \multirow[b]{2}{*}{ Label } & \multicolumn{3}{|c|}{ Fractional Coordinates } & \multirow{2}{*}{$\begin{array}{c}\text { Bader } \\
\text { Charge }\end{array}$} \\
\hline & & $\mathbf{x}$ & $\mathbf{y}$ & $\mathbf{Z}$ & \\
\hline S & S1 & 0.781452 & 0.117611 & 0.789712 & $+0.100 \mathrm{e}$ \\
\hline S & S2 & 0.248634 & 0.388050 & 0.713253 & $+0.087 \mathrm{e}$ \\
\hline S & $\mathrm{S} 3$ & 0.218548 & 0.882389 & 0.210288 & $+0.100 \mathrm{e}$ \\
\hline S & S4 & 0.751366 & 0.611950 & 0.286747 & $+0.087 \mathrm{e}$ \\
\hline S & S5 & 0.240214 & 0.156235 & 0.864964 & $-0.351 \mathrm{e}$ \\
\hline S & S6 & 0.786528 & 0.354636 & 0.644834 & $-0.354 \mathrm{e}$ \\
\hline S & S7 & 0.290649 & 0.627289 & 0.861732 & $-0.619 \mathrm{e}$ \\
\hline S & S8 & 0.781103 & 0.882764 & 0.633330 & $-0.635 \mathrm{e}$ \\
\hline S & S9 & 0.759786 & 0.843765 & 0.135036 & $-0.351 \mathrm{e}$ \\
\hline S & S10 & 0.213472 & 0.645364 & 0.355166 & $-0.354 \mathrm{e}$ \\
\hline $\mathrm{S}$ & S11 & 0.709351 & 0.372711 & 0.138268 & $-0.619 \mathrm{e}$ \\
\hline S & S12 & 0.218897 & 0.117237 & 0.366670 & $-0.635 \mathrm{e}$ \\
\hline $\mathrm{Li}$ & Li1 & 0.816462 & 0.119971 & 0.360895 & $+0.886 \mathrm{e}$ \\
\hline $\mathrm{Li}$ & Li2 & 0.313638 & 0.377167 & 0.145494 & $+0.886 \mathrm{e}$ \\
\hline $\mathrm{Li}$ & Li3 & 0.183539 & 0.880029 & 0.639105 & $+0.886 \mathrm{e}$ \\
\hline $\mathrm{Li}$ & Li4 & 0.686362 & 0.622833 & 0.854506 & $+0.886 \mathrm{e}$ \\
\hline
\end{tabular}


Table S8. Atom positions and corresponding Bader charges of solid state $\mathrm{Li}_{2} \mathrm{~S}_{7}$.

\begin{tabular}{cccccc}
\hline & & \multicolumn{3}{c}{ Fractional Coordinates } & Bader \\
Element & Label & $\mathbf{x}$ & $\mathbf{y}$ & $\mathbf{Z}$ & Charge \\
\hline S & S1 & 0.547354 & 0.024058 & 0.220653 & $-0.122 \mathrm{e}$ \\
S & S2 & 0.290683 & 0.253684 & 0.109818 & $-0.034 \mathrm{e}$ \\
S & S3 & 0.971936 & 0.941889 & 0.957983 & $-0.030 \mathrm{e}$ \\
S & S4 & 0.136975 & 0.100872 & 0.806038 & $-0.028 \mathrm{e}$ \\
S & S5 & 0.890489 & 0.524102 & 0.554756 & $-0.708 \mathrm{e}$ \\
S & S6 & 0.293299 & 0.929938 & 0.360874 & $-0.707 \mathrm{e}$ \\
S & S7 & 0.283753 & 0.760304 & 0.694902 & $-0.122 \mathrm{e}$ \\
Li & Li1 & 0.060280 & 0.388449 & 0.353441 & $+0.875 \mathrm{e}$ \\
Li & Li2 & 0.662590 & 0.989614 & 0.562025 & $+0.875 \mathrm{e}$ \\
\hline
\end{tabular}

Table S9. Atom positions and corresponding Bader charges of solid state $\mathrm{Li}_{2} \mathrm{~S}_{8}$.

\begin{tabular}{cccccc}
\hline & & \multicolumn{3}{c}{ Fractional Coordinates } & Bader \\
Element & Label & $\mathbf{X}$ & $\mathbf{y}$ & $\mathbf{Z}$ & Charge \\
\hline S & S1 & 0.639046 & 0.776793 & 0.891436 & $-0.044 \mathrm{e}$ \\
S & S2 & 0.386637 & 0.054605 & 0.299539 & $-0.082 \mathrm{e}$ \\
S & S3 & 0.237823 & 0.347482 & 0.987913 & $-0.013 \mathrm{e}$ \\
S & S4 & 0.607430 & 0.412584 & 0.939982 & $-0.095 \mathrm{e}$ \\
S & S5 & 0.794043 & 0.028658 & 0.356648 & $-0.095 \mathrm{e}$ \\
S & S6 & 0.940617 & 0.239088 & 0.591154 & $-0.710 \mathrm{e}$ \\
S & S7 & 0.459988 & 0.747543 & 0.645508 & $-0.725 \mathrm{e}$ \\
S & S8 & 0.306119 & 0.414628 & 0.232183 & $-0.012 \mathrm{e}$ \\
Li & Li1 & 0.446401 & 0.224044 & 0.623989 & $+0.884 \mathrm{e}$ \\
Li & Li2 & 0.925467 & 0.742485 & 0.600158 & $+0.890 \mathrm{e}$ \\
\hline
\end{tabular}




\section{Section SIII. Dynamic stabilities of polysulfide solid phases.}

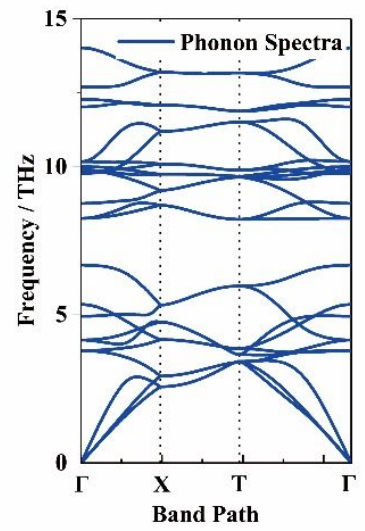

c.

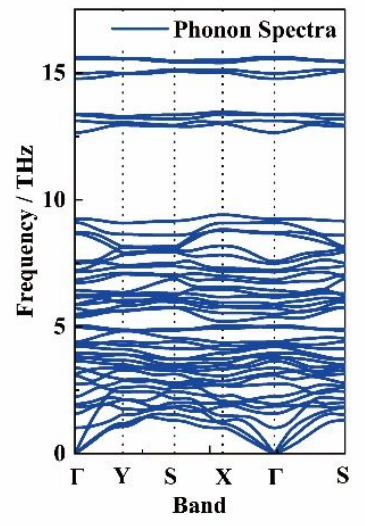

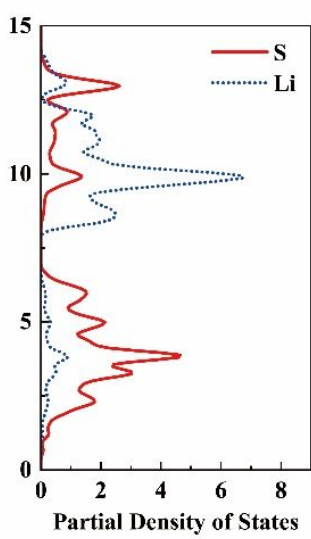

b.

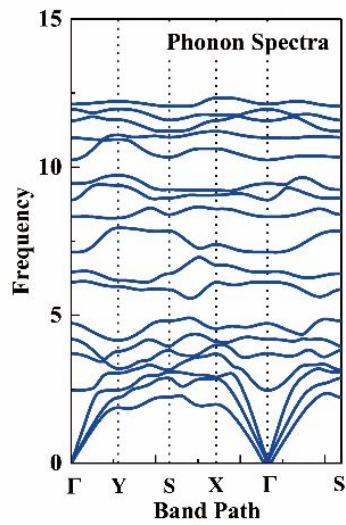

d.
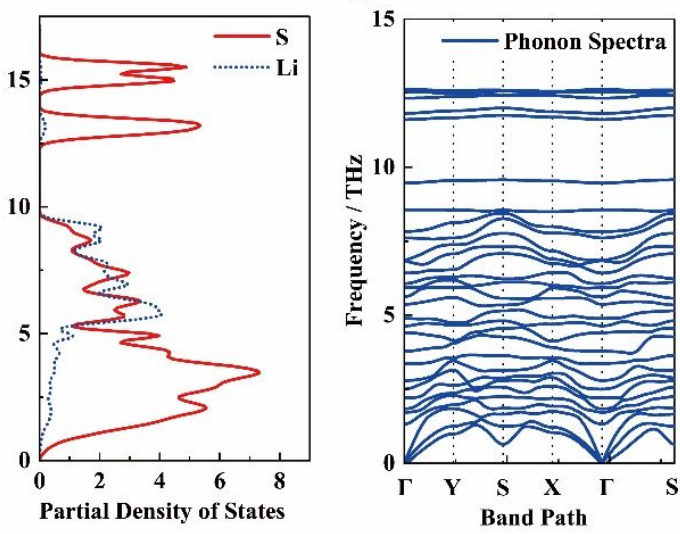
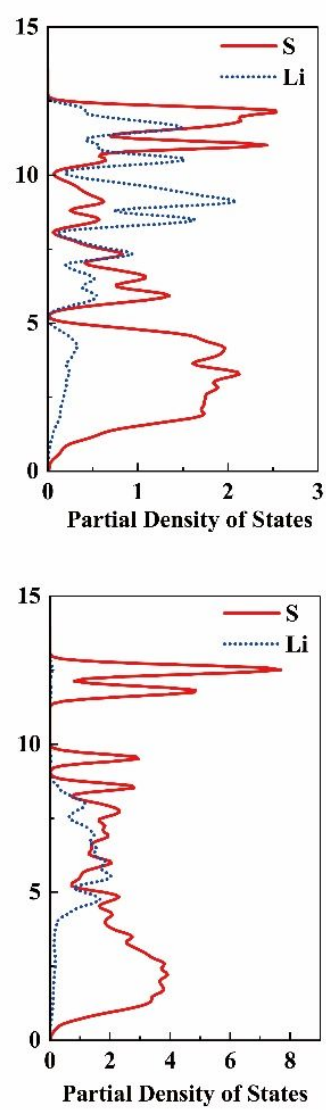

Figure S10. Calculated phonon spectra and partial density of states of a. solid state $\mathrm{Li}_{2} \mathrm{~S}_{2}$, b. solid state $\mathrm{Li}_{2} \mathrm{~S}_{4}$, c. solid state $\beta-\mathrm{Li}_{2} \mathrm{~S}_{6}$ and d. solid state $\mathrm{Li}_{2} \mathrm{~S}_{8}$. 
Section SIV. Electronic structures of polysulfide clusters and solid phases.

\section{1. $\operatorname{Li}_{2} S$}
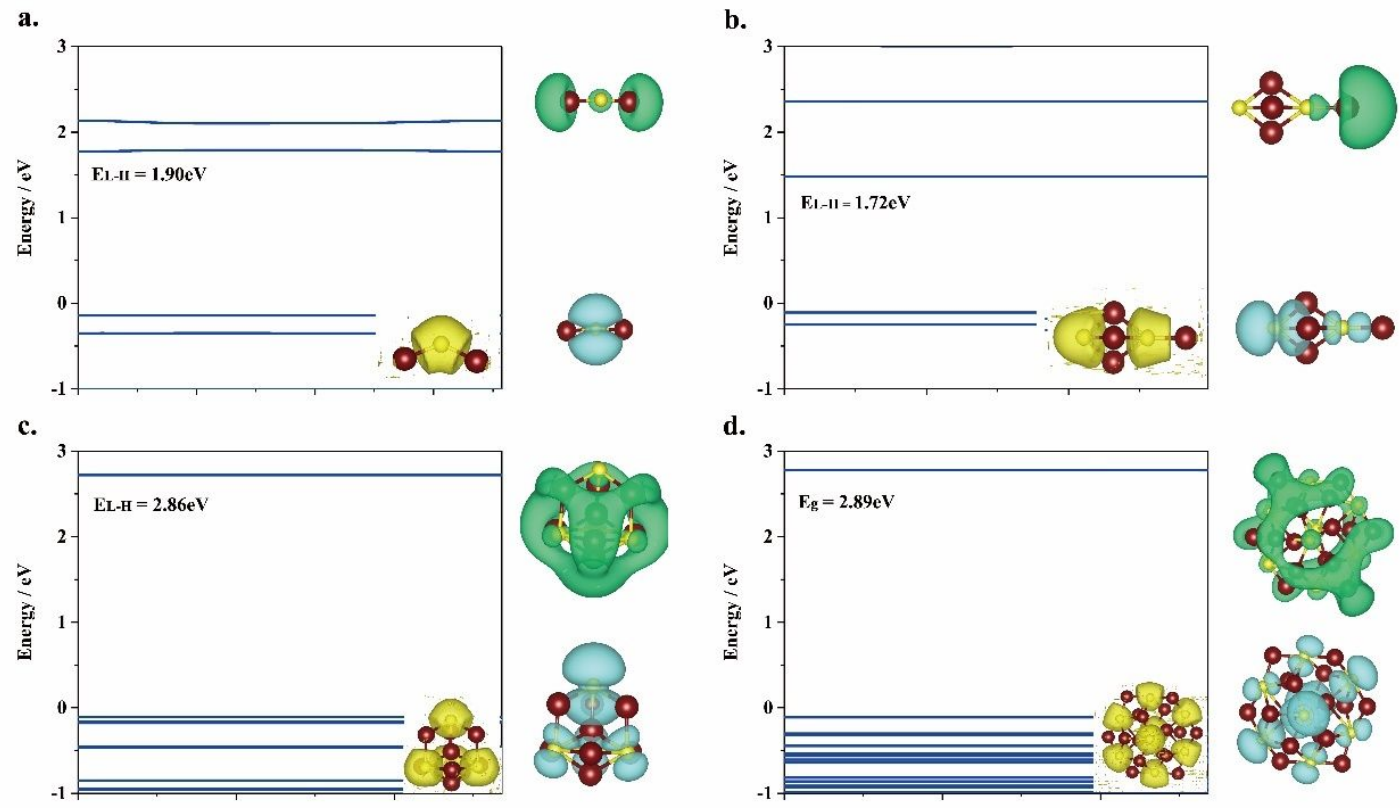

Figure S11. Calculated energy levels of a. $\mathrm{Li}_{2} \mathrm{~S}$, b. $\left(\mathrm{Li}_{2} \mathrm{~S}\right)_{2}$, c. $\left(\mathrm{Li}_{2} \mathrm{~S}\right)_{4}$ and d. $\left(\mathrm{Li}_{2} \mathrm{~S}\right)_{8}$, with ELF shown in the inset. Corresponding spatial charge distributions of LUMO and HOMO levels are presented in the right panel from top to bottom. 


\section{2. $\mathbf{L i}_{2} \mathbf{S}_{2}$}
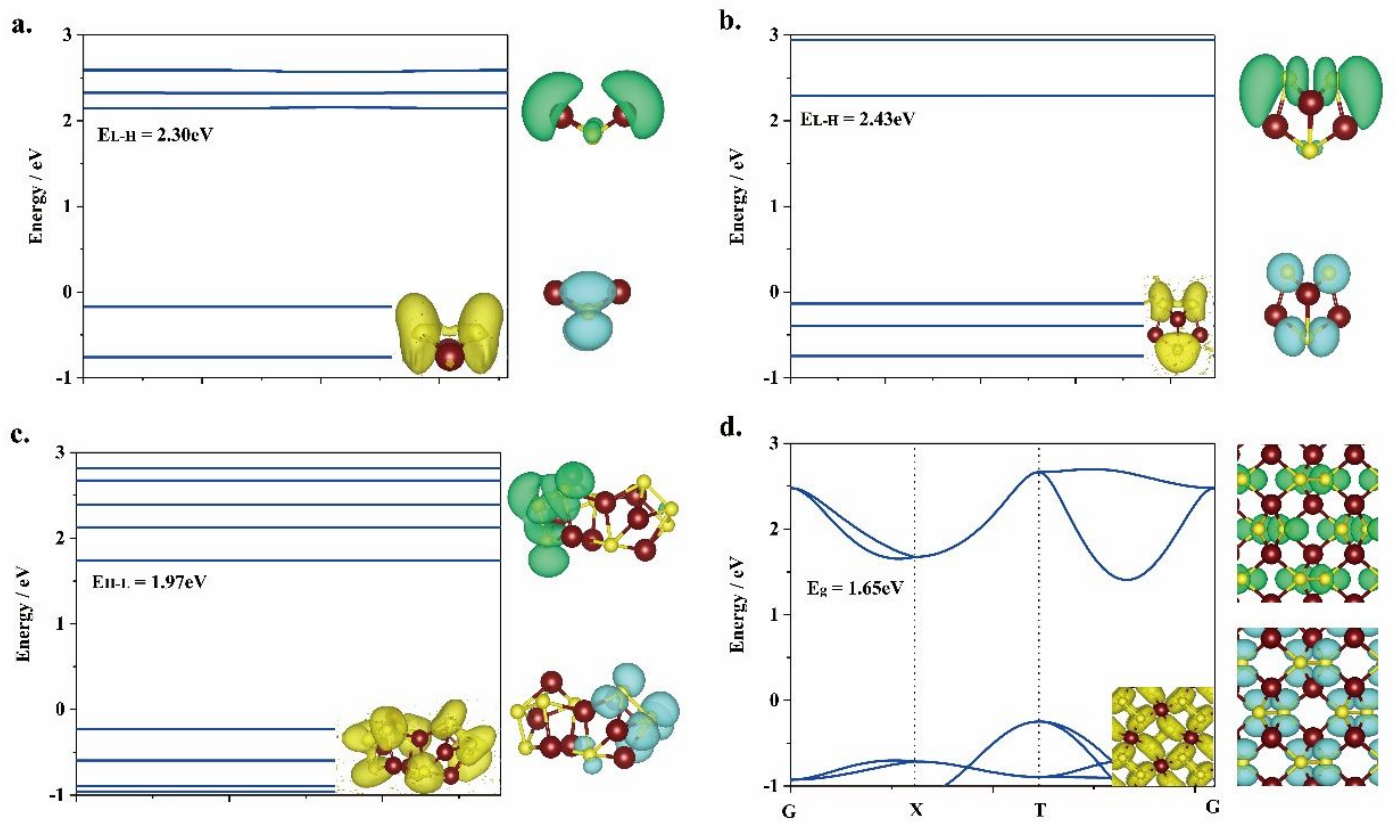

Figure S12. Calculated energy levels of a. $\mathrm{Li}_{2} \mathrm{~S}_{2}$, b. $\left(\mathrm{Li}_{2} \mathrm{~S}_{2}\right)_{2}$, c. $\left(\mathrm{Li}_{2} \mathrm{~S}_{2}\right)_{4}$ and d. solid phase $\mathrm{Li}_{2} \mathrm{~S}_{2}$, with ELF shown in the inset. Corresponding spatial charge distributions of LUMO and HOMO levels are presented in the right panel from top to bottom. 


\section{3. $\mathbf{L i}_{2} \mathrm{~S}_{3}$}

a.

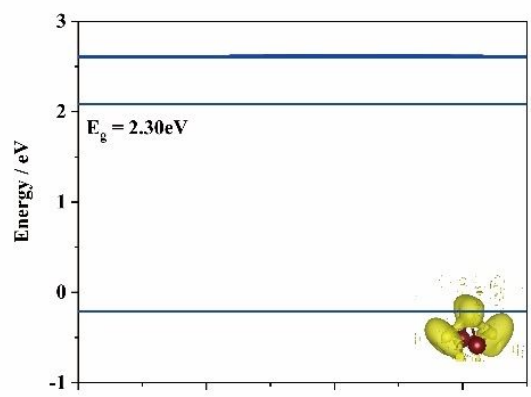

c.

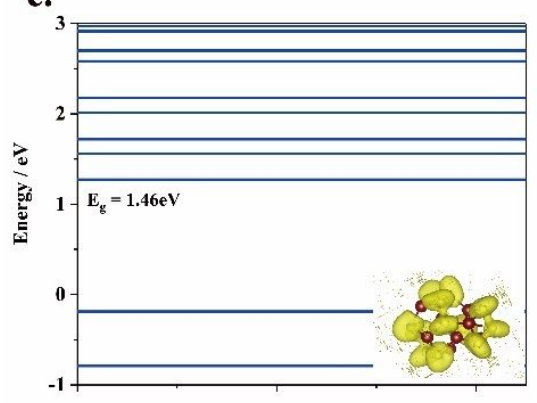

b.
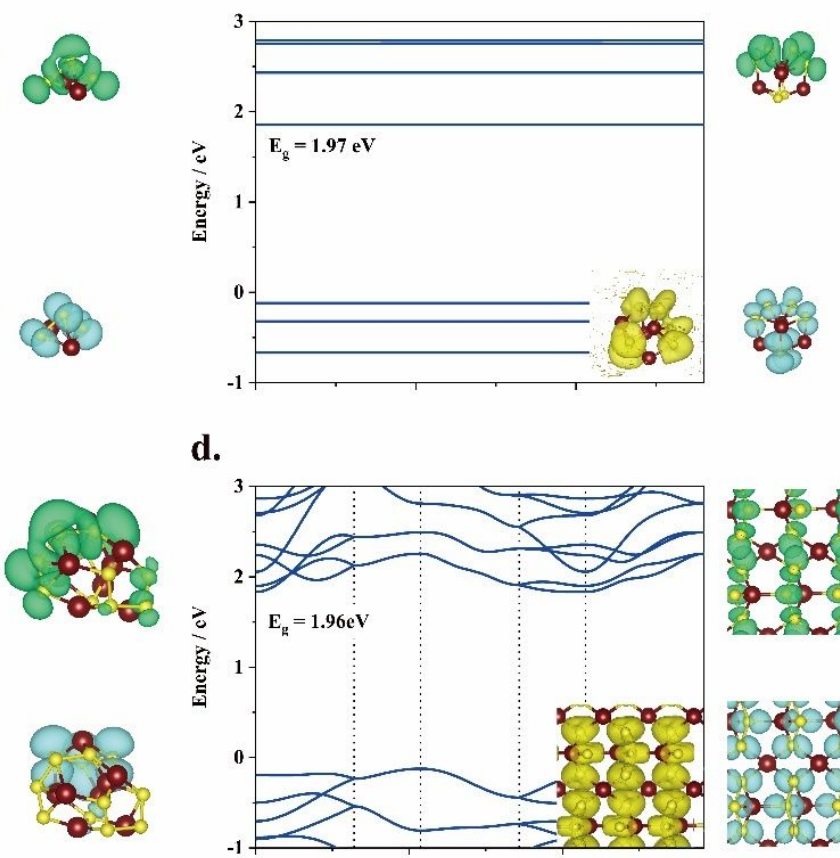

d.

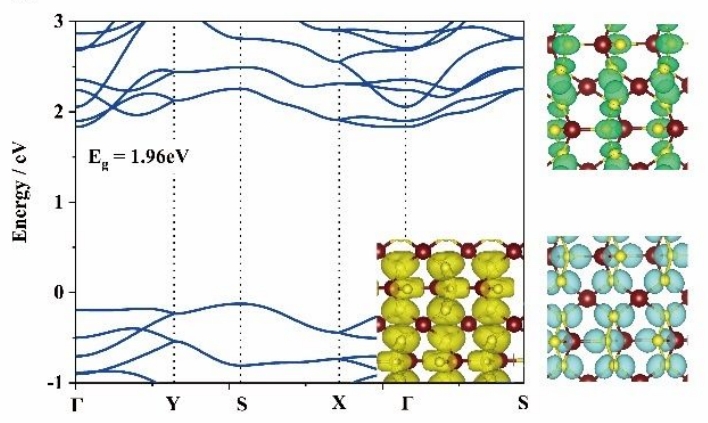

Figure S13. Calculated energy levels of a. $\mathrm{Li}_{2} \mathrm{~S}_{3}$, b. $\left(\mathrm{Li}_{2} \mathrm{~S}_{3}\right)_{2}$, c. $\left(\mathrm{Li}_{2} \mathrm{~S}_{3}\right)_{4}$ and d. solid phase $\mathrm{Li}_{2} \mathrm{~S}_{3}$, with ELF shown in the inset. Corresponding spatial charge distributions of LUMO and HOMO levels are presented in the right panel from top to bottom. 


\section{4. $\mathrm{Li}_{2} \mathrm{~S}_{4}$}
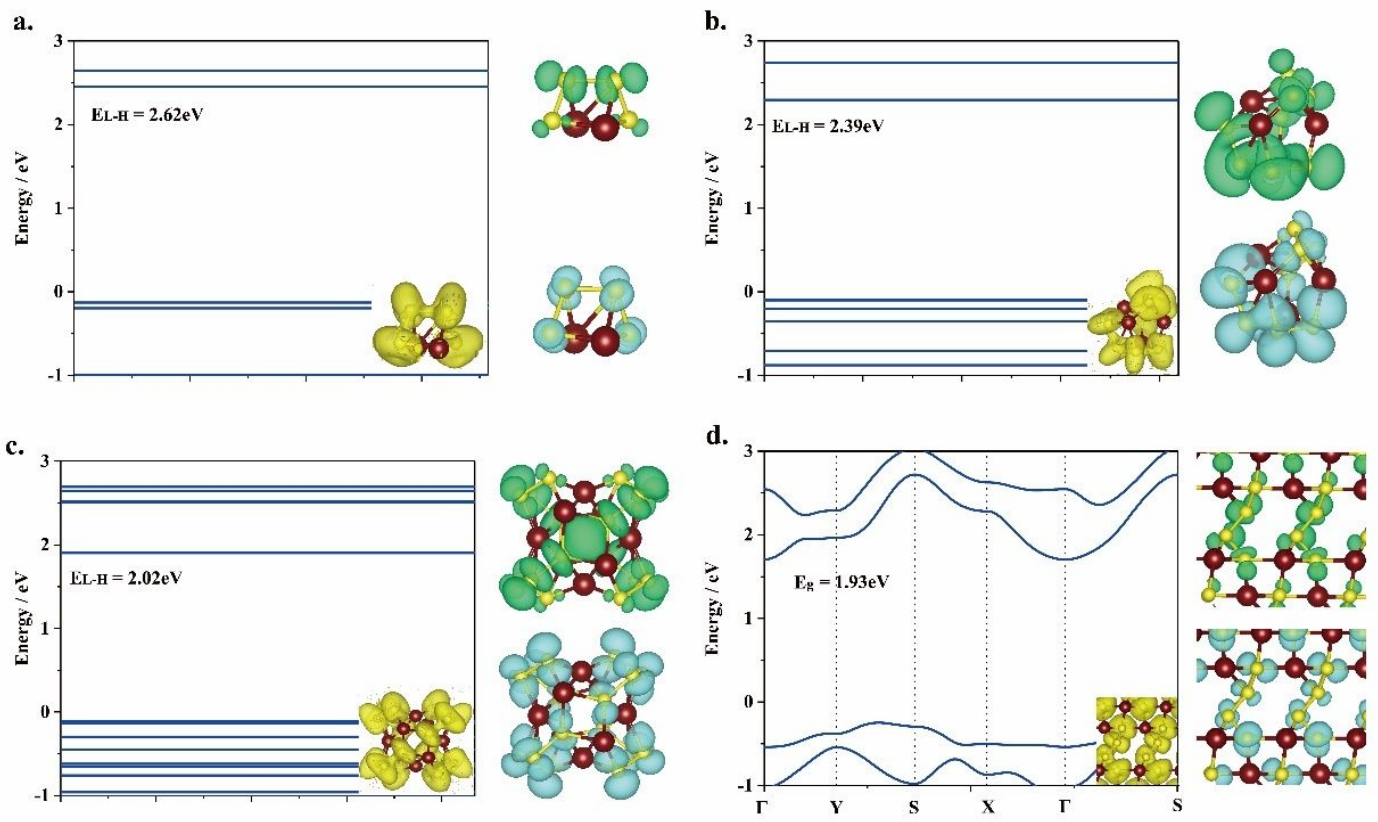

Figure S14. Calculated energy levels of a. $\mathrm{Li}_{2} \mathrm{~S}_{4}$, b. $\left(\mathrm{Li}_{2} \mathrm{~S}_{4}\right)_{2}$, c. $\left(\mathrm{Li}_{2} \mathrm{~S}_{4}\right)_{4}$ and d. solid phase $\mathrm{Li}_{2} \mathrm{~S}_{4}$, with ELF shown in the inset. Corresponding spatial charge distributions of LUMO and HOMO levels are presented in the right panel from top to bottom. 


\section{5. $\mathrm{Li}_{2} \mathrm{~S}_{6}$}
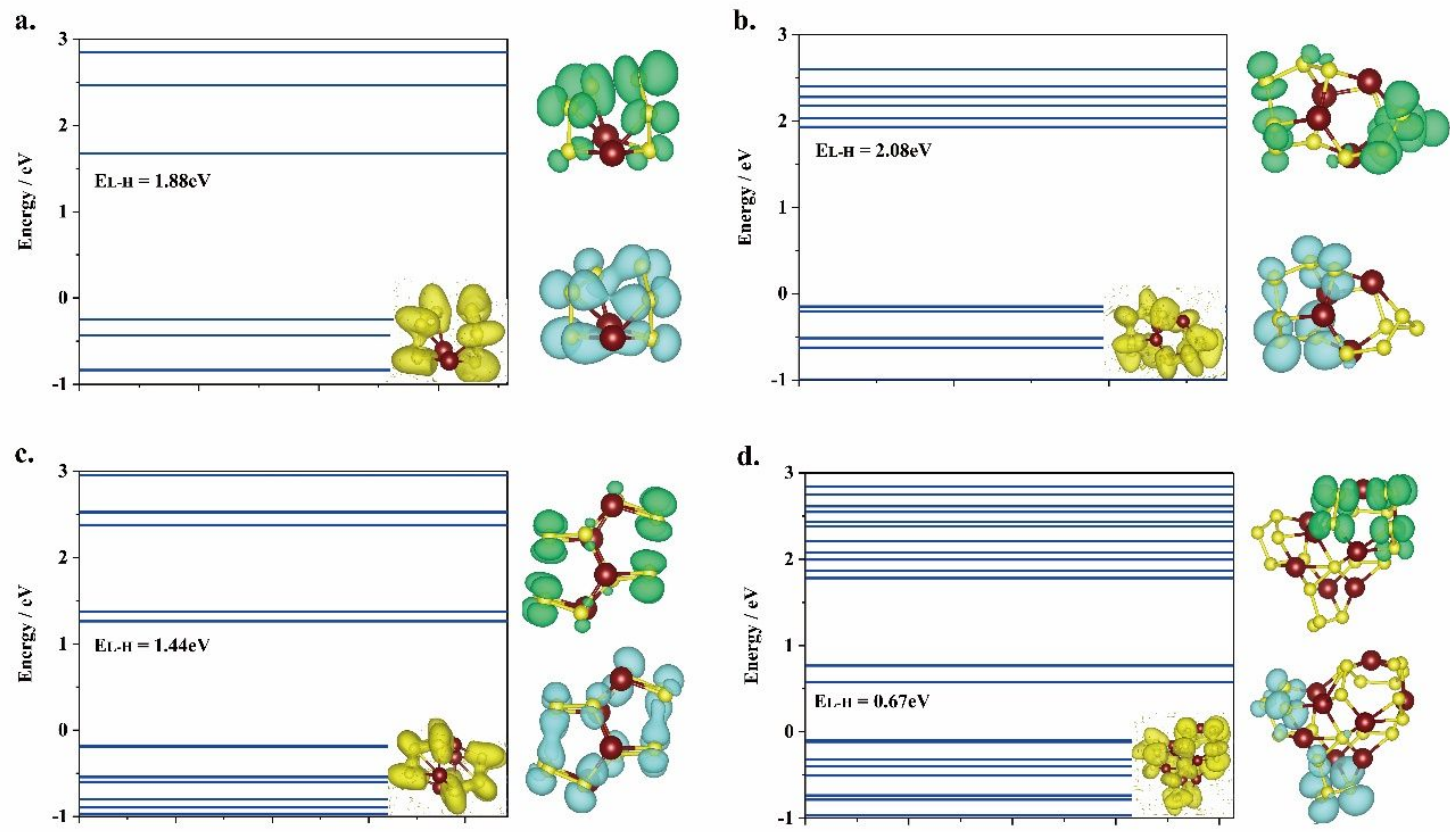

Figure S15. Calculated energy levels of a. $\mathrm{Li}_{2} \mathrm{~S}_{6}$, b. $\alpha-\left(\mathrm{Li}_{2} \mathrm{~S}_{6}\right)_{2}$, c. $\beta-\left(\mathrm{Li}_{2} \mathrm{~S}_{6}\right)_{2}$ and $\mathrm{d}$. $\left(\mathrm{Li}_{2} \mathrm{~S}_{6}\right)_{4}$, with ELF shown in the inset. Corresponding spatial charge distributions of LUMO and HOMO levels are presented in the right panel from top to bottom. 

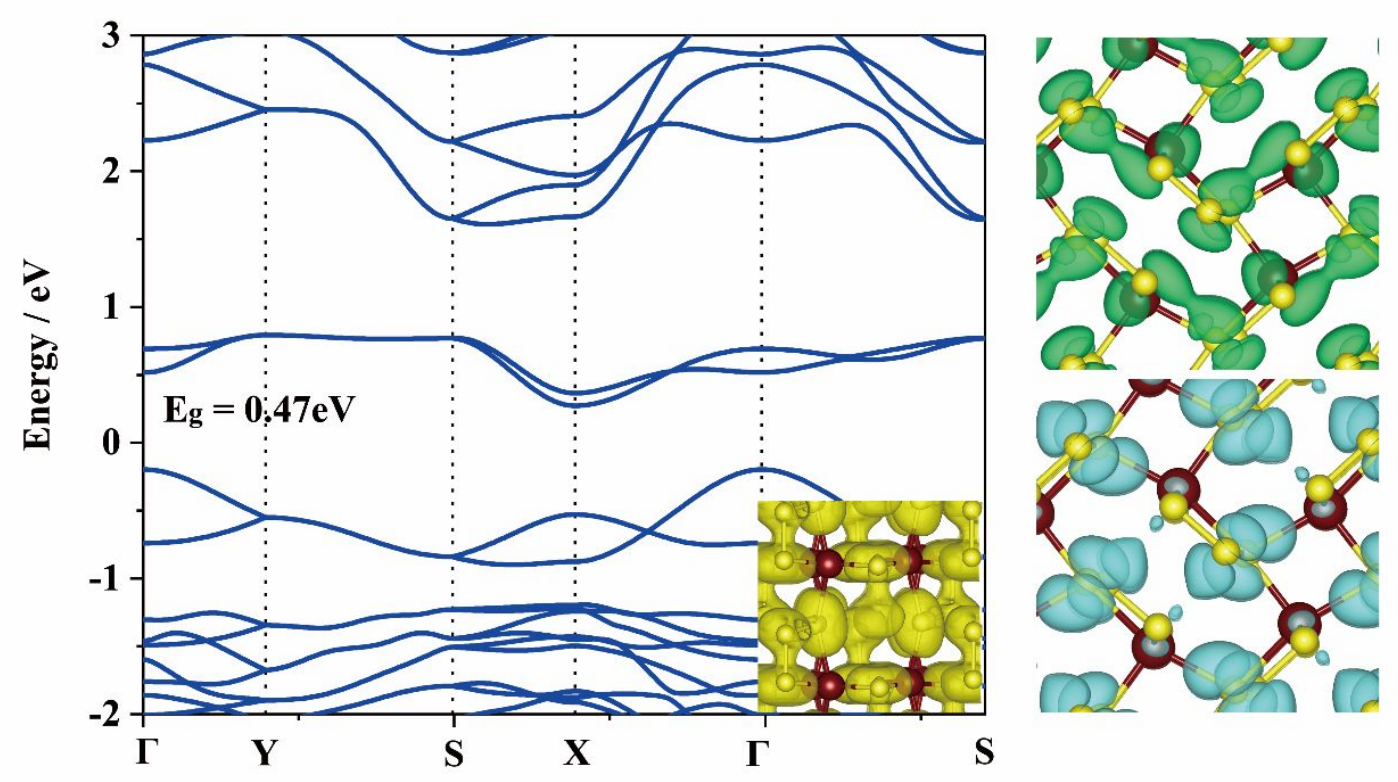

Figure S16. Calculated band structure of $\alpha-\mathrm{Li}_{2} \mathrm{~S}_{6}$ based on PBE scheme, with ELF shown in the inset. Corresponding spatial charge distributions of conduction band minimum $(\mathrm{CBM})$ and valance band maximum $(\mathrm{VBM})$ are presented in the right panel from top to bottom.

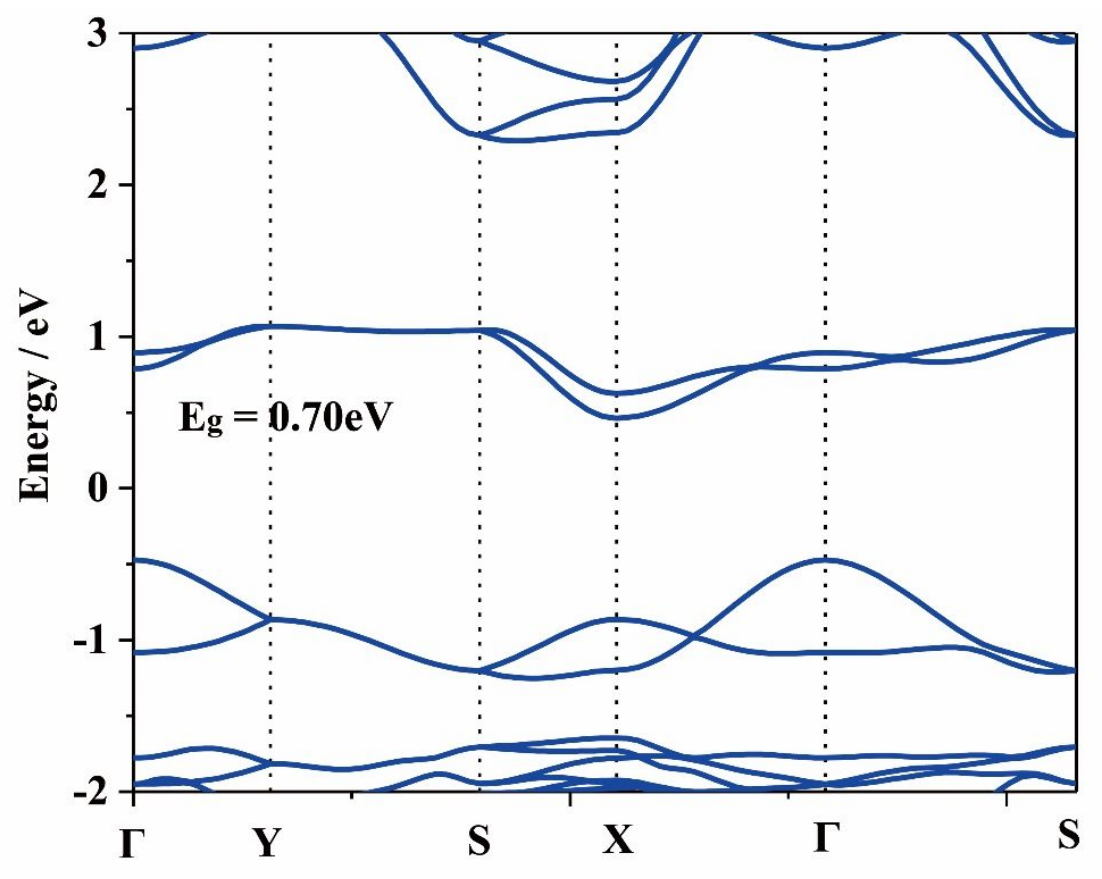

Figure S17. Calculated band structure of $\alpha-\mathrm{Li}_{2} \mathrm{~S}_{6}$ based on HSE scheme, with band gap being corrected to $0.70 \mathrm{eV}$. 


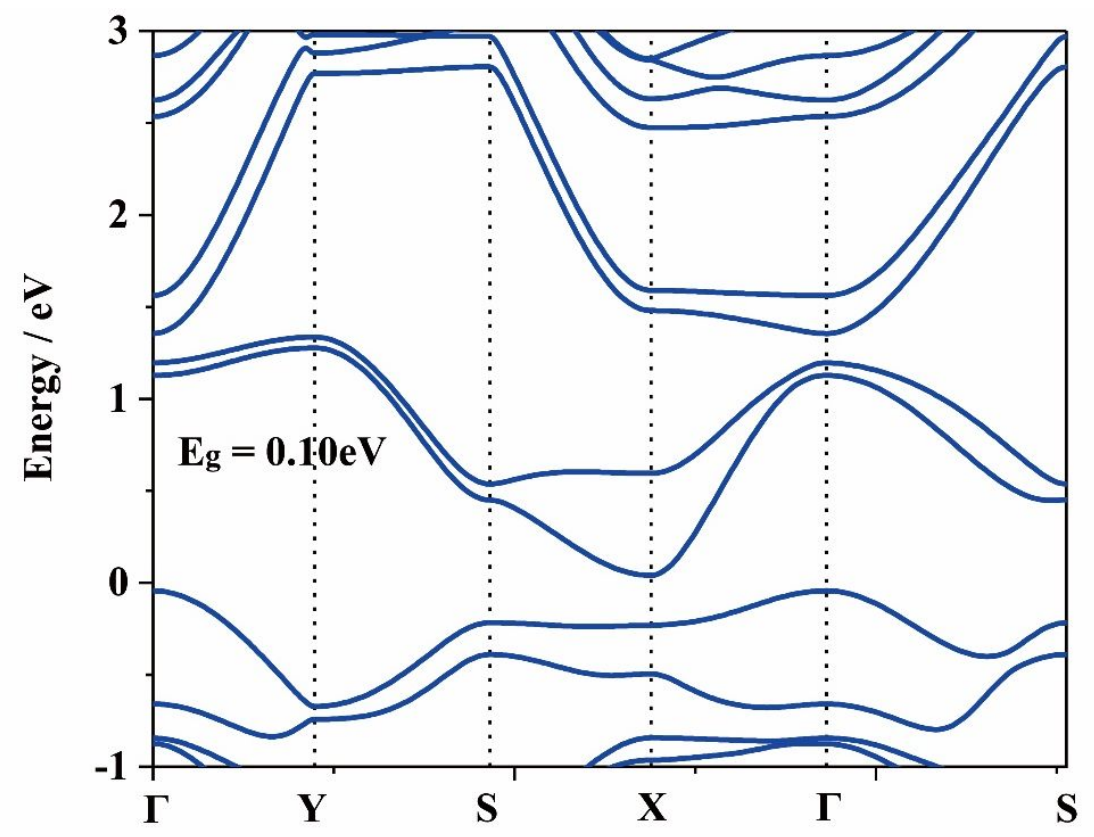

Figure S18. Calculated band structure of $\beta-\mathrm{Li}_{2} \mathrm{~S}_{6}$ based on PBE scheme, with band gap as low as $0.10 \mathrm{eV}$. 


\section{6. $\mathrm{Li}_{2} \mathrm{~S}_{8}$}

a.

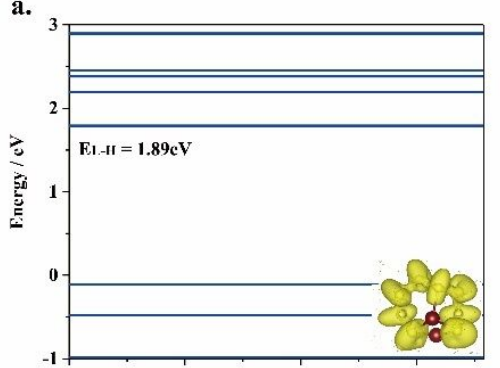

c.

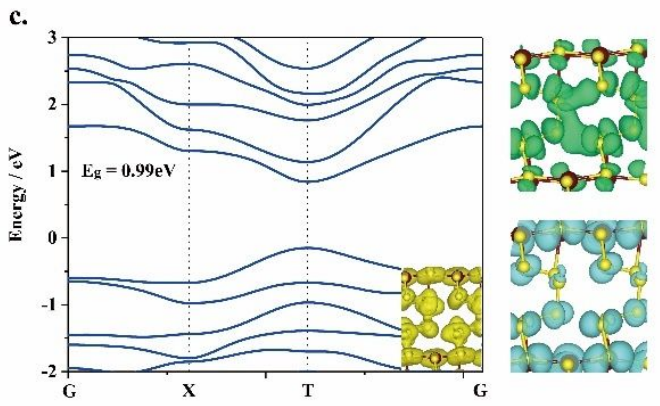

b.
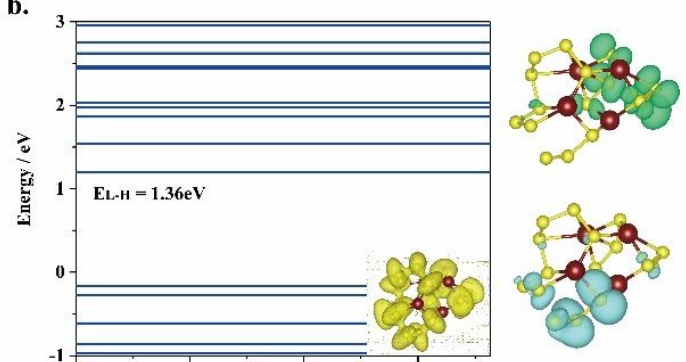

d.
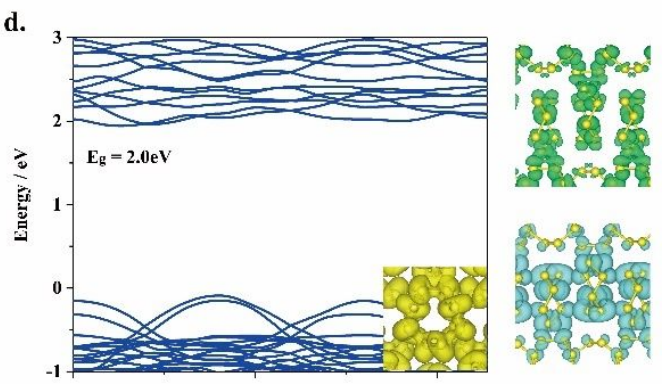

Figure S19. Calculated energy levels of a. $\mathrm{Li}_{2} \mathrm{~S}_{8}$, b. $\left(\mathrm{Li}_{2} \mathrm{~S}_{8}\right)_{2}$, c. solid phase $\mathrm{Li}_{2} \mathrm{~S}_{8}$ and d. solid phase $\alpha-S_{8}$, with ELF shown in the inset. Corresponding spatial charge distributions of LUMO and HOMO levels are presented in the right panel from top to bottom. 
Section V. Dissolved polysulfides in DOL and DME electrolyte

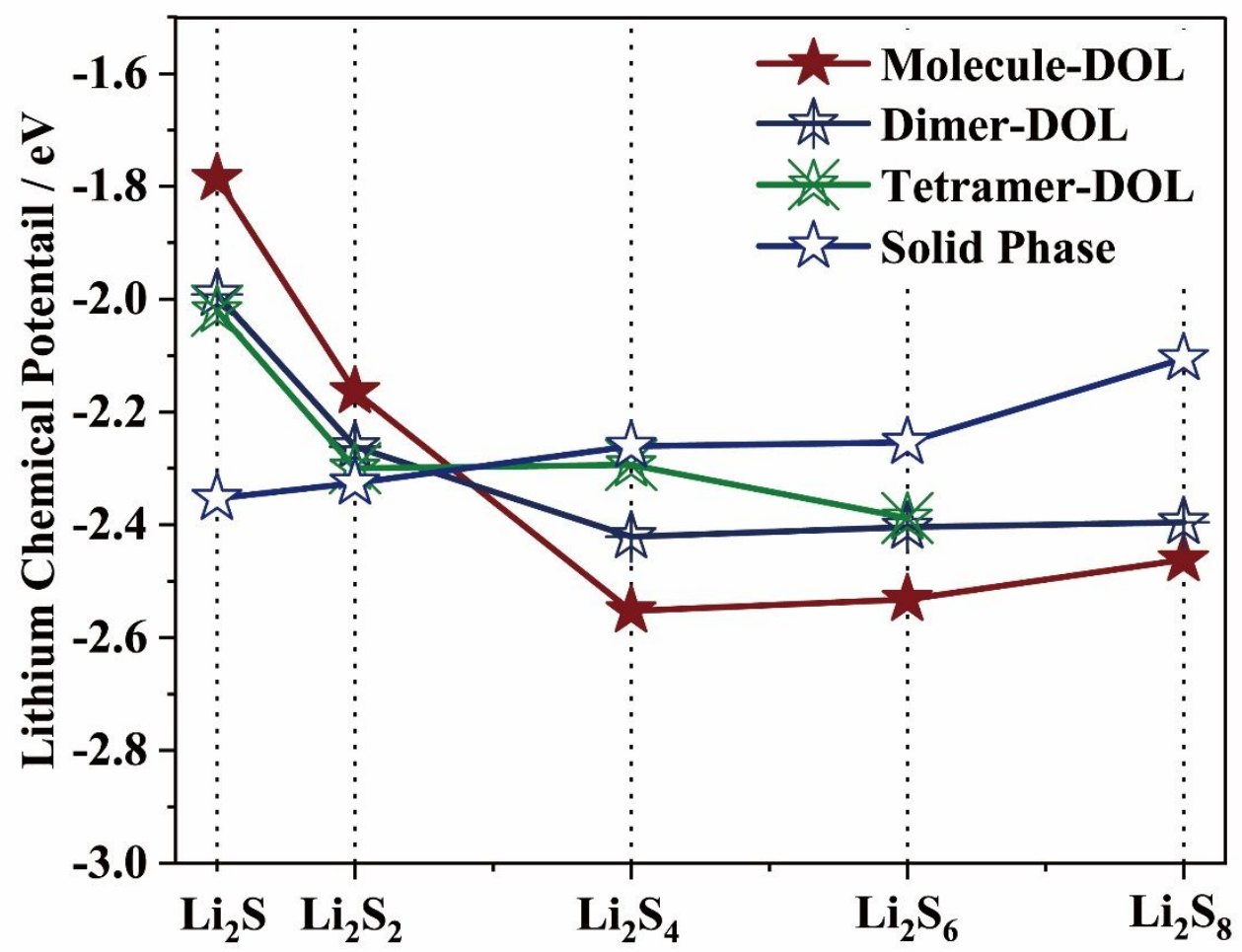

Figure S20. Lithium chemical potential of polysulfide clusters dissolved in DOL electrolyte and solid phases.

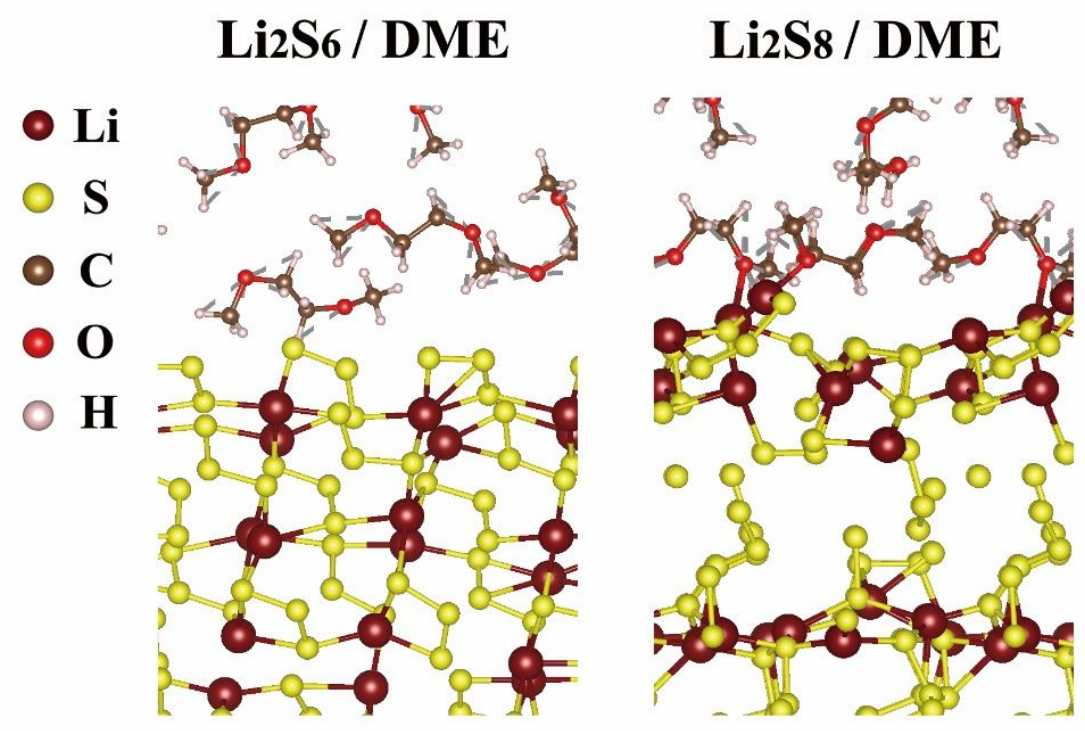

Figure S21. AIMD simulations for $\mathrm{DME} / \mathrm{Li}_{2} \mathrm{~S}_{6}$ and $\mathrm{DME} / \mathrm{Li}_{2} \mathrm{~S}_{8}$ interfaces. 


\section{Section VI. Simulated Raman Spectra}

In the following figures, intensity of Raman spectra is denoted as: $\mathrm{m}=$ medium, $\mathrm{s}=$ strong, vs $=$ very strong.

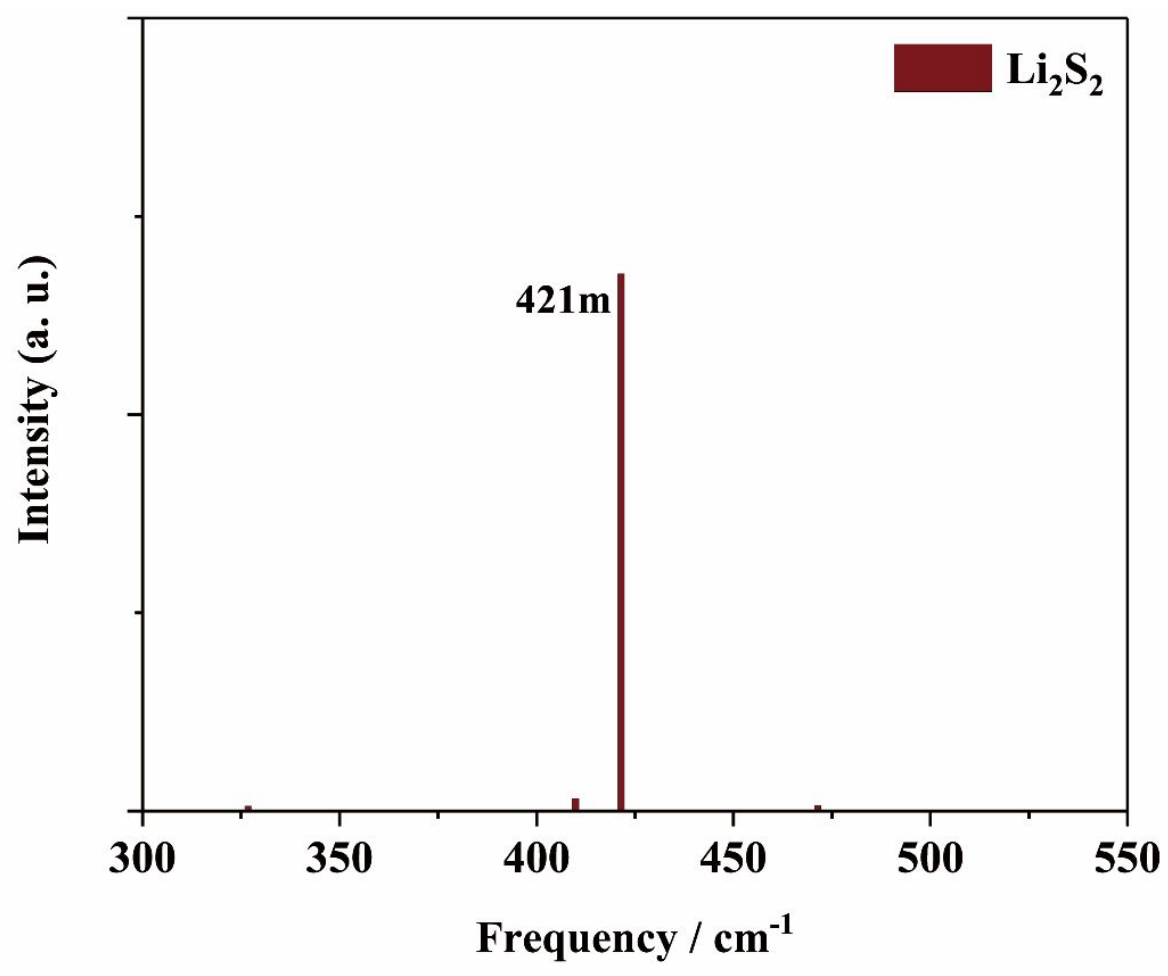

Figure S22. Simulation of Raman spectra of solid state $\mathrm{Li}_{2} \mathrm{~S}_{2}$. 


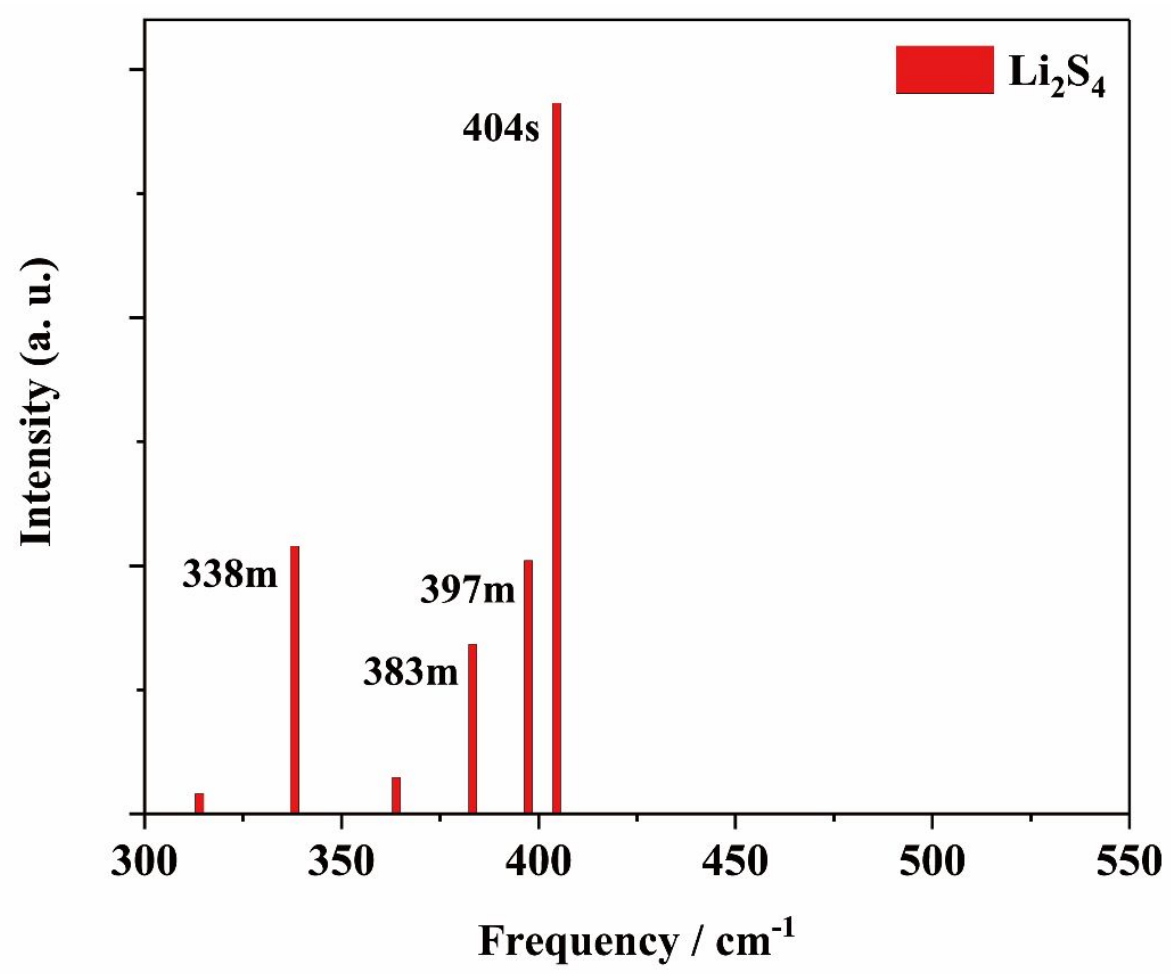

Figure S23. Simulation of Raman spectra of solid state $\mathrm{Li}_{2} \mathrm{~S}_{4}$.

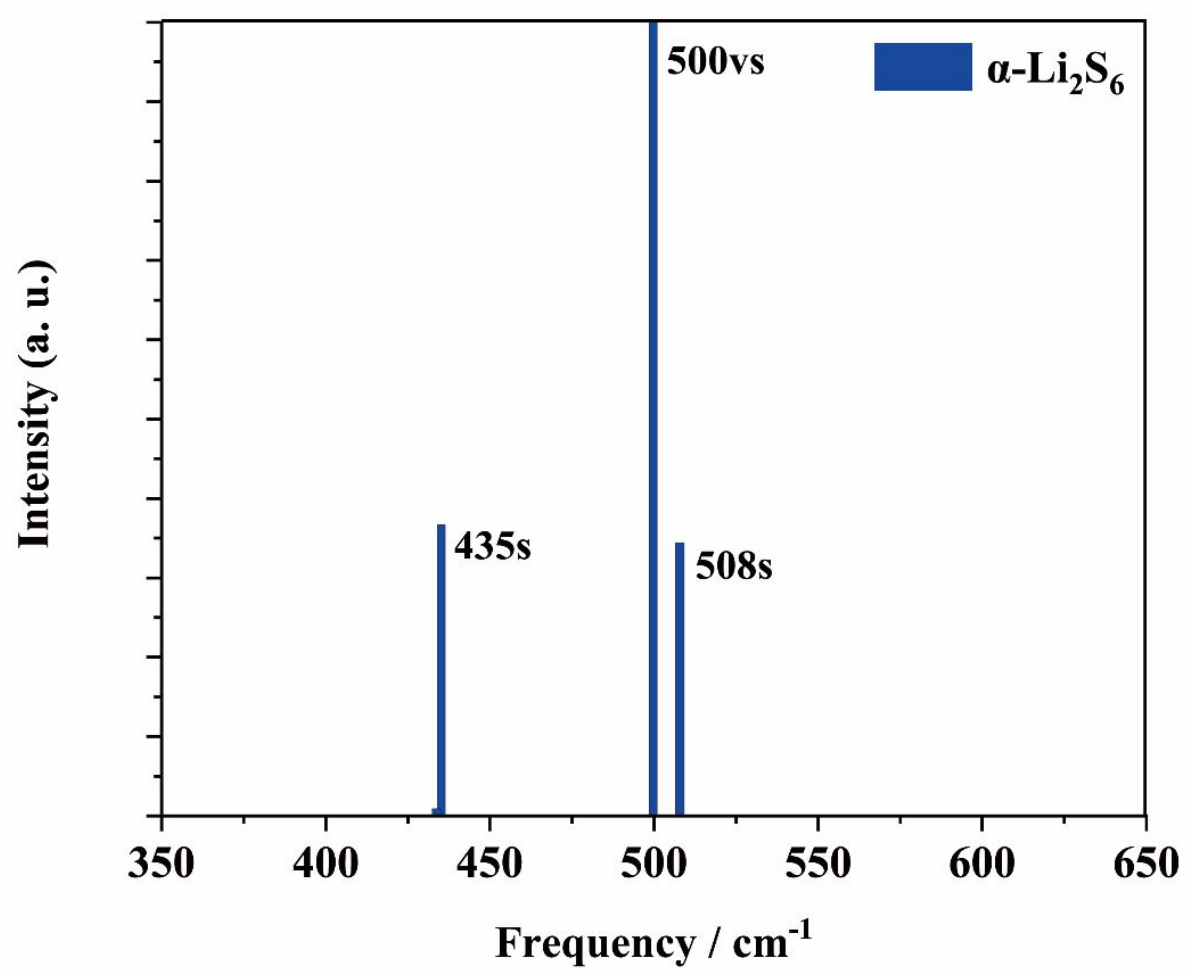

Figure S24. Simulation of Raman spectra of solid state $\alpha-\mathrm{Li}_{2} \mathrm{~S}_{6}$. 


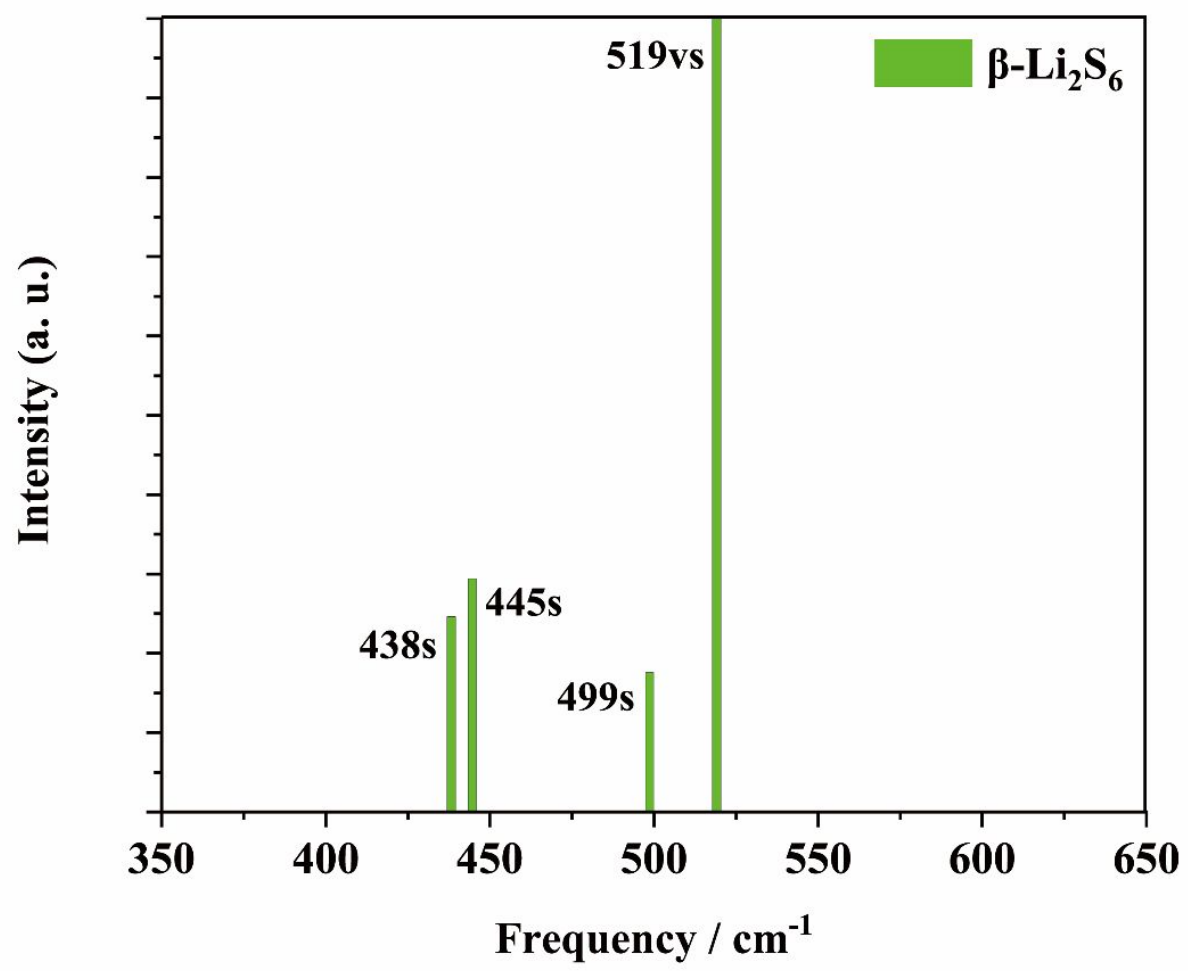

Figure S25. Simulation of Raman spectra of solid state $\beta-\mathrm{Li}_{2} \mathrm{~S}_{6}$.

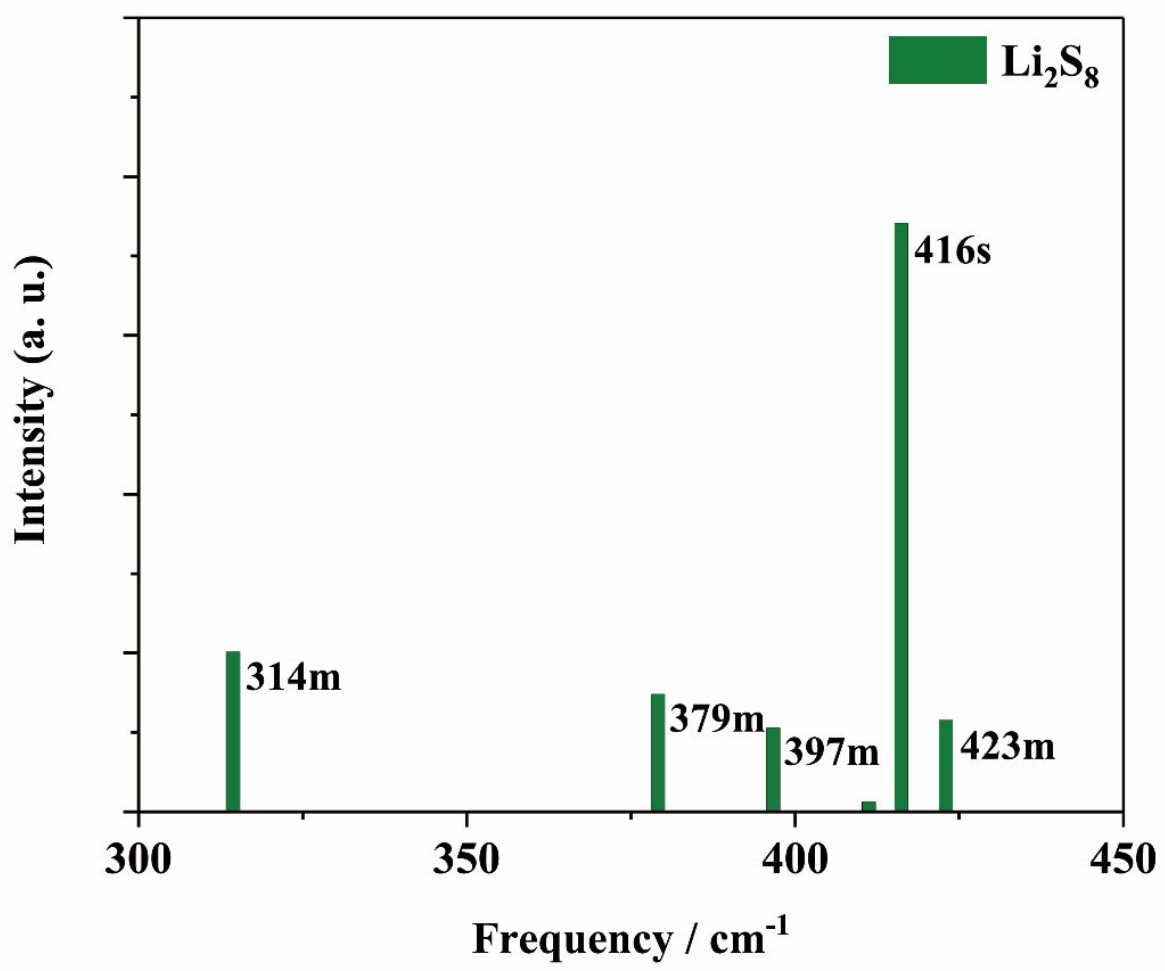

Figure S26. Simulation of Raman spectra of solid state $\mathrm{Li}_{2} \mathrm{~S}_{8}$. 


\section{Section SVII. Binding Strength of Graphene towards Polysulfides}

Resolved binding strength is applied here and defined as:

$$
E_{b}=\frac{E_{\left(L i_{2} S\right)_{m}}+L M-E_{L M}}{m}-E_{L i_{2} S_{x}}^{0}
$$

Where $E_{\left(L i_{2} S\right)_{m}}+L M$ and $E_{L M}$ denotes the total energy of the adsorption system and loading materials as graphene. $\left(L i_{2} S\right)_{m}(\mathrm{~m}=1,2,4,8$ and slab) represents molecules, dimers, tetramers, octamers and slabs, respectively. While $E_{L i_{2} S}^{0}$ corresponds to the energy of freestanding $\mathrm{Li}_{2} \mathrm{~S}$ molecule. Therefore, resolved binding strength reflects two factors that determine stable states of polysulfides: external interaction with loading materials and internal cohesive interaction among themselves.

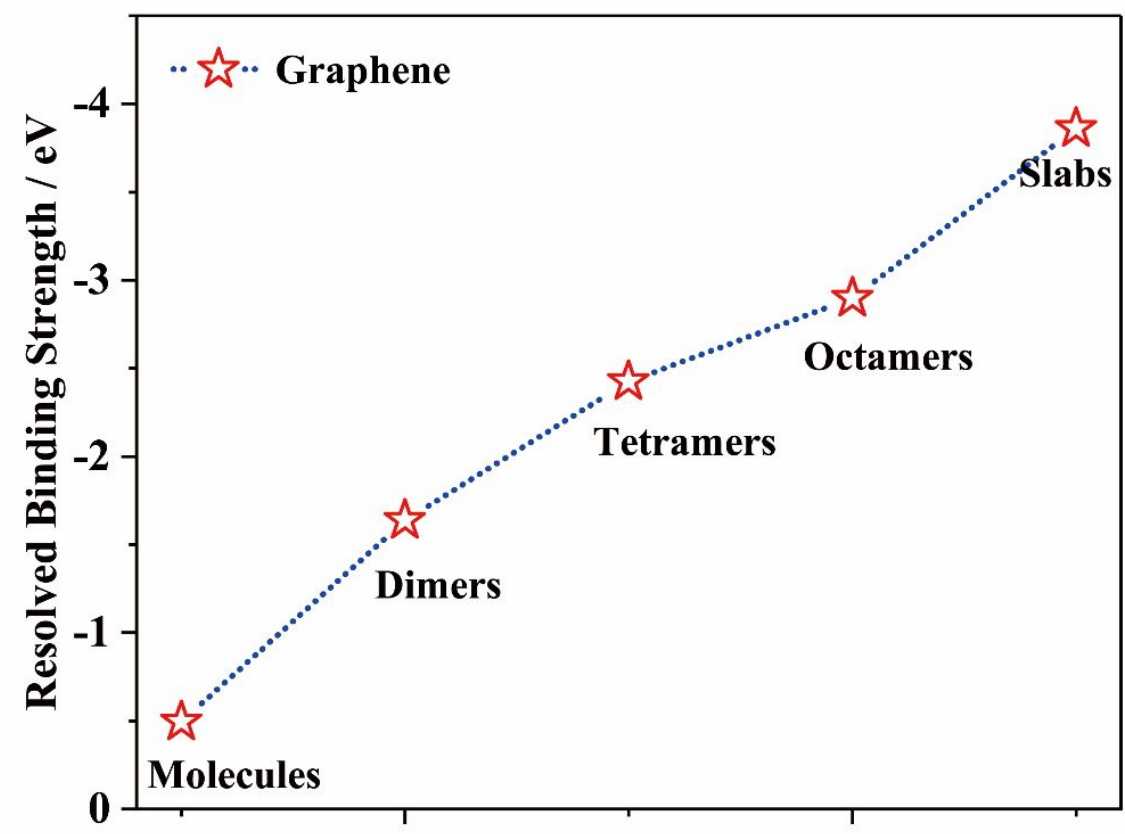

Figure S27. Evolution of resolved binding strength for polysulfide species adsorbed on graphene. 


\section{Reference}

(1) Kresse, G.; Furthmuller, J. Comput. Mater. Sci. 1996, 6, 15.

(2) Kresse, G.; Furthmuller, J. Phys. Rev. B 1996, 54, 11169.

(3) Blochl, P. E. Phys. Rev. B 1994, 50, 1758.

(4) Kresse, G.; Joubert, D. Phys. Rev. B 1999, 59, 1758.

(5) Perdew, J. P.; Burke, K.; Ernzerhof, M. Phys. Rev. Lett. 1996, 77, 3865.

(6) Monkhorst, H. J.; Pack, J. D. Phys. Rev. B 1976, 13, 5188.

(7) Paier, J.; Marsman, M. M.; Hummer, K.; Kresse, G.; Gerber, I. C.; Angyan, J. G.

J. Chem. Phys. 2006, 124, 154709.

(8) Krukau, A. V.; Vydrov, O. A.; Izmaylov, A. F.; Scuseria, G. E. J. Chem. Phys. 2006, $125,224106$.

(9) Henkelman, G.; Arnaldsson, A.; Jonsson, H. Comput. Mater. Sci. 2006, 36, 354.

(10) Tang, W.; Sanville, E.; Henkelman, G. J. Phys.: Condens. Matter 2009, $21,084204$.

(11) Dronskowski, R.; Blochl, P. E. J. Phys. Chem. 1993, 97, 8617-8624.

(12) Deringer, V. L.; Tchougreeff, A. L.; Dronskowski, R. J. Phys. Chem. A 2011, 115, $5461-5466$.

(13) Maintz, S.; Deringer, V. L.; Tchougreeff, A. L.; Dronskowski, R. J. Comput. Chem. 2013, 34, 2557.

(14) Maintz, S.; Deringer, V. L.; Tchougreeff, A. L.; Dronskowski, R. J. Comput. Chem. 
2016, 37, 1030 .

(15) Park, H.; Koh, H. S.; Siegel, D. J. J. Phys. Chem. C 2015, 119, 4675-4683.

(16) Lee, K.; Murray, E. D.; Kong, L.; Lundqvist, B. I.; Langreth, D. C. Phys. Rev. B 2011, $82,081101$.

(17) Klimes J.; Bowler D. R.; Michelides, A. Phys. Rev. B 2011, 83, 195131.

(18) Dion D.; Rydberg H.; Schroder E.; Langreth D. C.; Lundqvist, B. I. Phys. Rev. Lett. 2004, 92, 246401.

(19) Hamada, I. Phys. Rev. B 2014, 89, 121103.

(20) Jain, A.; Ong, S. P.; Hautler, G.; Chen, W.; Richards, W. D.; Dacek, S.; Cholia, S.; Gunter, D.; Skinner, D.; Ceder, G.; Persson, K. A. APL Materials 2013, 1, 011002. (21) Ong, S. P.; Wang, L.; Kang, B.; Ceder, G. Chem. Mater. 2008, 20, 1798.

(22) Jain, A.; Hautier, G.; Ong, S. P.; Moore, C.; Fischer, C.; Persson, K.; Ceder, G. Phys. Rev. B 2011, 84, 045115.

(23) Ong, S. P.; Richards, W. D.; Jain, A.; Hautier, G.; Kocher, M.; Cholia, S.; Gunter, D.; Chevrier, V. L.; Persson, K.; Ceder, G. Comput. Mater. Sci. 2013, 68, 314.

(24) Wang, L.; Maxisch, T.; Ceder, G. Phys. Rev. B 2006, 73, 195107.

(25) Grindy, S.; Meredig, B.; Kirklin, S.; Saal, J. E.; Wolverton, C. Phys. Rev. B 2013, 87,075150 .

(26) Kresse, G.; Furthmuller, J.; Hafner, J. Europhys. Lett. 1995, 32, 729.

(27) Wang, Y.; Lv, J.; Zhu, L.; Ma, Y. Comput. Phys. Commun. 2012, 183, 2063.

(28) Kresse, G.; Hafner, J. Phys. Rev. B: Condens. Matter Mater. Phys. 1993, 47, 558. 
(29) Kresse, G.; Hafner, J. Phys. Rev. B. Condens. Matter Mater. Phys. 1994, 49, 14251.

(30) Henkelman, G.; Uberuaga, B. P.; Jónsson, H. J. Chem. Phys. 2000, 113, 9901.

(31) Wasalathiake, K. C.; Roknuzzaman, M.; Ostrikov, K.; Ayoko, G. A.; Yan, C. RSC $A d v$. 2018, 8, 2271-2279.

(32) Yoon, C.; Kim, D.; Park, I.; Chang, D.; Kim, B.; Lee, B.; Oh, K.; Kang, K. Adv. Funct. Mater. 2017, 27, 1702887. 